\title{
AN ANALYTIC APPROACH TO SPECTRAL FLOW IN VON NEUMANN ALGEBRAS
}

\author{
M-T. BENAMEUR, A. L. CAREY, J. PHILliPS, A. RENNIE, F. A. SUKOCHEV, AND K. P. \\ WOJCIECHOWSKI
}

\begin{abstract}
The analytic approach to spectral flow is about ten years old. In that time it has evolved to cover an ever wider range of examples. The most critical extension was to replace Fredholm operators in the classical sense by Breuer-Fredholm operators in a semifinite von Neumann algebra. The latter have continuous spectrum so that the notion of spectral flow turns out to be rather more difficult to deal with. However quite remarkably there is a uniform approach in which the proofs do not depend on discreteness of the spectrum of the operators in question. The first part of this paper gives a brief account of this theory extending and refining earlier results. It is then applied in the latter parts of the paper to a series of examples. One of the most powerful tools is an integral formula for spectral flow first analysed in the classical setting by Getzler and extended to Breuer-Fredholm operators by some of the current authors. This integral formula was known for Dirac operators in a variety of forms ever since the fundamental papers of Atiyah, Patodi and Singer. One of the purposes of this exposition is to make contact with this early work so that one can understand the recent developments in a proper historical context. In addition we show how to derive these spectral flow formulae in the setting of Dirac operators on (non-compact) covering spaces of a compact spin manifold using the adiabatic method. This answers a question of Mathai connecting Atiyah's $L^{2}$-index theorem to our analytic spectral flow. Finally we relate our work to that of Coburn, Douglas, Schaeffer and Singer on Toeplitz operators with almost periodic symbol. We generalise their work to cover the case of matrix valued almost periodic symbols on $\mathbf{R}^{N}$ using some ideas of Shubin. This provides us with an opportunity to describe the deepest part of the theory namely the semifinite local index theorem in noncommutative geometry. This theorem, which gives a formula for spectral flow was recently proved by some of the present authors. It provides a far-reaching generalisation of the original 1995 result of Connes and Moscovici.
\end{abstract}

\section{INTRODUCTION}

Spectral flow ${ }^{1}$ is normally associated with paths of operators with discrete spectrum such as Dirac operators on compact manifolds. ${ }^{2}$ Even then it is only in the last decade that analytic definitions have been introduced (previously the definitions were topological). Recently it has been discovered that if one takes an analytic approach to spectral flow then one can handle examples where the operators may have zero in the continuous spectrum.

\footnotetext{
${ }^{1}$ MSC Subject classification: Primary: 19K56, 58J20; secondary: 46L80, 58J30.

${ }^{2}$ This research is supported by the ARC (Australia), NSERC (Canada) and an early career grant from the University of Newcastle.
} 
The aim of this article is to give a discussion of spectral flow in as general an analytic setting as is currently feasible. In fact we consider unbounded operators affiliated to a semifinite von Neumann algebra and give examples where the phenomenon of spectral flow for paths of such operators occurs quite naturally. There has been a lot published recently on this subject, which is rather technical although the ideas can be explained reasonably simply. This article is thus partly a review of this theory aimed at exposing these recent results to a wider audience. As the early papers dealt with von Neumann algebras with trivial centre (factors) and the more general situation of non-trivial centre was only recently completely understood we also felt that it was timely to collect the basic definitions and results in one place. Moreover we have rounded out the account with some additional new results and some carefully chosen illustrative examples.

The methods we use are motivated by noncommutative geometry however our results may be stated without using that language. The novel feature of spectral flow for operators affiliated to a general semifinite von Neumann algebra is that the operators in question may have zero in their continuous spectrum. It is thus rather surprising that spectral flow can even be defined in this situation.

We focus on spectral flow for a continuous path of self adjoint unbounded Breuer-Fredholm operators $\left\{D(s)=D_{0}+A(s)\right\}$ for $s \in[0,1]$ in the sense that $A(s)$ is a norm continuous family of bounded self adjoint operators in a fixed semifinite von Neumann algebra $\mathcal{N}$ and $D(s)$ is affiliated to $\mathcal{N}$ for all $s \in[0,1]$ (we will elaborate on all of this terminology in subsequent Sections). We restrict to the paths of bounded perturbations because the analytic theory is complete and many interesting examples exist. The wider question of paths where the domain and the Hilbert space $H(s)$, on which $D(s)$ is densely defined, varies with $s$ is still under investigation (see the article by Furutani [39] for motivation). This situation may arise on manifolds with boundary where one varies the metric and is a difficult problem unless one makes very specific assumptions. An approach to this question has been introduced by Leichtnam and Piazza [45] building on ideas of Dai and Zhang [33] which in turn is based on unpublished work of F. Wu. It works for Dirac type operators in both the case of closed manifolds and the case of (possibly noncompact) covering spaces. This new notion is that of spectral section.

Spectral sections enable one to define spectral flow as an invariant in the K-theory of a certain $C^{*}$-subalgebra of the von Neumann algebras that we consider in this article. We have chosen not to discuss it here because, although it can handle the case where the space $H(s)$ varies with $s$ we feel that the theory is not yet in final form. Moreover it reduces in the von Neumann setting to Phillips approach. Another omission is a discussion of the topological meaning of spectral flow in the general analytic setting. We refer the reader to the work of Getzler [40, Booß-Bavnbek et al [9], and Lesch [47].

While our aim is to put in one place all of the basic ideas we do not include complete proofs instead referring where necessary to the literature. Thus we start with a summary of Fredholm theory in a general semifinite von Neumann algebra $\mathcal{N}$ with a fixed faithful semifinite trace $\tau$. We refer to such operators as ' $\tau$-Breuer-Fredholm' because we can trace the origins of the theory to Breuer [11, 12] but we need to refine his theory to take account of non-uniqueness of the trace $\tau$ on a von Neumann algebra with non-trivial centre. In this setting we discuss Phillips' 
analytic approach to spectral flow for paths of bounded self adjoint Breuer-Fredholm operators in $\mathcal{N}$. Then we include some simple analytic examples that show the theory is non-trivial.

The theory for paths $\{D(s)\}_{s \in[0,1]}$ of self adjoint unbounded operators proceeds via the map $s \rightarrow D(s) \rightarrow D(s)\left(1+D(s)^{2}\right)^{-1 / 2}$. When $\{D(s)\}$ is a norm-continuous path of perturbations (of the kind considered above) of $D(0)$, an unbounded self adjoint $\tau$-Breuer-Fredholm operator, then its image under this map is a continuous path in the space of bounded self-adjoint $\tau$ Breuer-Fredholm operators [15]. Although (in the case $\mathcal{N}=\mathcal{B}(\mathcal{H})$ ) spectral flow can be defined directly for such paths of unbounded operators [9], we can also define spectral flow in terms of the corresponding path of bounded self-adjoint operators.

The second half of the paper is about analytic formulae for spectral flow that have appeared in the literature. After reviewing these formulae we relate them to classical theory via a study of spectral flow of generalised Dirac operators on compact manifolds without boundary and their covering spaces. A question first raised by Mathai [4] is settled by relating spectral flow to the $L^{2}$ index theorem. The deepest result in the theory is the semifinite local index theorem which we illustrate by application to an example of spectral flow for differential operators with almost periodic coefficients. This is inspired by work of Shubin 62] who initiated this line of enquiry. The generalisation to semifinite von Neumann algebras of the local index theorem of Connes and Moscovici 32 was achieved in papers of some of the present authors [18, 19] and has other interesting applications (not included here) for example see Pask et al [54].

\section{Preliminaries}

2.1. Notation. Our basic reference for von Neumann algebras is Dixmier 34] where many of the concepts we discuss here are described in detail. For the theory of ideals of compact operators in a semifinite von Neumann algebra we refer to Fack et al [38] and Dodds et al 35. Throughout this paper we will consider $\mathcal{N}$, a semifinite von Neumann algebra (of type $I_{\infty}$ or $I I_{\infty}$ or mixed type) acting on a separable Hilbert space $\mathcal{H}$. We will denote by $\tau$ a fixed faithful, normal semifinite trace on $\mathcal{N}$ (with the usual normalization if $\mathcal{N}$ is a type $I_{\infty}$ factor). The norm-closed 2-sided ideal in $\mathcal{N}$ generated by the projections of finite trace (usually called $\tau$-finite projections) will be denoted by $\mathcal{K}_{\tau \mathcal{N}}$ or just $\mathcal{K}_{\mathcal{N}}$ to lighten the notation. The quotient algebra $\mathcal{N} / \mathcal{K}_{\mathcal{N}}$ will be denoted by $\mathcal{Q}_{\mathcal{N}}$ and will be called the (generalized) Calkin algebra. We will let $\pi$ denote the quotient mapping $\mathcal{N} \rightarrow \mathcal{Q}_{\mathcal{N}}$.

We will let $\mathcal{F}$ denote the space of all $\tau$-Breuer-Fredholm operators in $\mathcal{N}$, i.e. ,

$$
\mathcal{F}=\left\{T \in \mathcal{N} \mid \pi(T) \text { is invertible in } \mathcal{Q}_{\mathcal{N}}\right\} .
$$

We denote by $\mathcal{F}^{s a}$ the space of self adjoint operators in $\mathcal{F}$. The more interesting part of the space of self adjoint $\tau$-Breuer-Fredholm operators in $\mathcal{N}$ will be denoted by $\mathcal{F}_{*}^{s a}$, i.e.,

$$
\mathcal{F}_{*}^{s a}=\left\{T \in \mathcal{F} \mid T=T^{*} \text { and } \pi(T) \text { is neither positive nor negative }\right\} .
$$

2.2. Some history. For $\mathcal{N}$ being the algebra of bounded operators on $\mathcal{H}$, i.e. the type $I_{\infty}$ factor case, Atiyah and Lusztig [2, 3] have defined the spectral flow of a continuous path in $\mathcal{F}_{*}^{s a}$ to be the number of eigenvalues (counted with multiplicities) which pass through 0 in the positive direction minus the number which pass through 0 in the negative direction as one moves 
from the initial point of the path to the final point. This definition is appealing geometrically as an "intersection number" and has been made precise [40, 10, 57 although it cannot easily be generalised beyond the type $I_{\infty}$ factor. Other motivating remarks may be found in BooßBavnbek et al [8, 9. More importantly, there is no obvious generalization of this definition if the algebra $\mathcal{N}$ is of type $I I_{\infty}$, where the spectrum of a self-adjoint Breuer-Fredholm operator is not discrete in a neighbourhood of zero. J. Kaminker has described this as the problem of counting "moving globs of spectrum".

In his 1993 Ph.D. thesis, V.S. Perera [55, 56] gave a definition of the spectral flow of a loop in $\mathcal{F}_{*}^{s a}$ for a $I I_{\infty}$ factor, $\mathcal{N}$. He showed that the space, $\Omega\left(\mathcal{F}_{*}^{s a}\right)$, of loops based at a unitary $(2 P-1)$ in $\mathcal{F}_{*}^{s a}$, is homotopy equivalent to the space, $\mathcal{F}$, of all Breuer-Fredholm operators in the $I I_{\infty}$ factor, $P \mathcal{N} P$. Since Breuer [11, 12] showed that the index map $\mathcal{F} \longrightarrow \mathbf{R}$ classifies the connected components of $\mathcal{F}$, Perera defines spectral flow as the composition $s f: \Omega\left(\mathcal{F}_{*}^{s a}\right) \longrightarrow \mathcal{F} \longrightarrow \mathbf{R}$ and so obtains the isomorphism $\pi_{1}\left(\mathcal{F}_{*}^{s a}\right) \cong \mathbf{R}$. He also showed that this gives the "heuristically correct" answer for a simple family of loops.

While this is an important and elegant result, it has a couple of weaknesses. Firstly, since the map $s f$ is not defined directly and constructively on individual loops it is not clear why spectral flow is counting "moving globs of spectrum". Secondly, in the nonfactor setting where the von Neumann algebra may have summands of finite type the map may not extend to paths which are not loops in any sensible way: in a finite algebra (see 5.1) there can be paths with nonzero spectral flow, but every loop has zero spectral flow.

Phillips' approach [57, 58] is the following. Let $\chi$ denote the characteristic function of the interval $[0, \infty)$. If $\left\{B_{t}\right\}$ is any continuous path in $\mathcal{F}_{*}^{s a}$, then $\left\{\chi\left(B_{t}\right)\right\}$ is a discontinuous path of projections whose discontinuities arise precisely because of spectral flow. For example, if $t_{1}<t_{2}$ are neighbouring path parameters and if the projections $P_{i}=\chi\left(B_{t_{i}}\right)$ commute, then the spectral flow from $t_{1}$ to $t_{2}$ should be trace $\left(P_{2}-P_{1} P_{2}\right)$ minus $\operatorname{trace}\left(P_{1}-P_{1} P_{2}\right)(=$ amount of nonnegative spectrum gained minus amount of nonnegative spectrum lost). However, this is clearly the index of the operator $P_{1} P_{2}: P_{2}(H) \rightarrow P_{1}(H)$. If these projections do not commute then one can still make sense of this index provided $\pi\left(P_{1}\right)=\pi\left(P_{2}\right)$ in the Calkin algebra. This notion was called essential codimension by Brown, Douglas and Fillmore [13] in the type $I_{\infty}$ case and denoted by $e c\left(P_{1}, P_{2}\right)$. Perera [55, 56] defined the obvious extension of this concept to $I I_{\infty}$ factors and used it to explain why his definition of spectral flow gives the "right" answer in a representative family of simple loops. Phillips' [58, 57] new ingredient is the fact that the operator $P_{1} P_{2}: P_{2}(H) \rightarrow P_{1}(H)$ is always a $\tau$-Breuer-Fredholm operator provided $\left\|\pi\left(P_{1}\right)-\pi\left(P_{2}\right)\right\|<1$. While Phillips only proved this in the case of a factor, we observed in Carey et al [21] that it works for a general semifinite von Neumann algebra. We will explain the proof in the next Section, and show that the condition $\left\|\pi\left(P_{1}\right)-\pi\left(P_{2}\right)\right\|<1$ is necessary and sufficient for $P_{1} P_{2}$ to be $\tau$-Breuer-Fredholm.

Since we can (easily) show that the mapping $t \mapsto \pi\left(\chi\left(B_{t}\right)\right)$ is continuous, we can partition the parameter interval $a=t_{0}<t_{1}<\cdots<t_{k}=b$ so that on each small subinterval the 
projections $\pi\left(\chi\left(B_{t}\right)\right)$ are all close. Letting $P_{i}=\chi\left(B_{t_{i}}\right)$ for $i=0,1, \cdots, k$ we then define:

$$
s f\left(\left\{B_{t}\right\}\right)=\sum_{i=1}^{k} \operatorname{Ind}\left(P_{i-1} P_{i}\right) .
$$

With a little effort this works equally well in both the type $I_{\infty}$ and $I I_{\infty}$ settings and agrees with all previous definitions of spectral flow where they exist. A simple lemma is the key to showing that $s f$ is well-defined and (path-) homotopy invariant. Defining $\operatorname{Hom}\left(\mathcal{F}_{*}^{s a}\right)$ to be the homotopy groupoid of $\mathcal{F}_{*}^{s a}$, Phillips proved the following theorem in the case of a factor. It extends to the general semifinite case [19].

Theorem 2.1. If $\mathcal{N}$ is a general semifinite von Neumann algebra then sf as defined above is a homomorphism from $\operatorname{Hom}\left(\mathcal{F}_{*}^{s a}\right)$ to $\mathbf{R}$ which restricts to an isomorphism of $\pi_{1}\left(\mathcal{F}_{*}^{\text {sa }}\right)$ with $\mathbf{Z}$ (respectively $\mathbf{R}$ ) when $\mathcal{N}$ is a factor of type $I_{\infty}$ (respectively, type $I I_{\infty}$ ).

We note that to show that $s f$ is one-to-one on $\pi_{1}\left(\mathcal{F}_{*}^{s a}\right)$ one must rely on Perera's result that $\Omega\left(\mathcal{F}_{*}^{s a}\right) \simeq \mathcal{F}$. We also remark that in paragraphs 7,8 and 9 of the introduction to the Atiyah-Patodi-Singer paper [3] the authors appear to be hinting at the existence of a notion of spectral flow (for paths of self-adjoint Breuer-Fredholm operators in a $I I_{\infty}$ factor) to be used as a possible tool in an alternate proof of their index theorem for flat bundles. In some sense this hope is realised by the generalisation [18, 19] of the Connes-Moscovici local index formula to the semifinite von Neumann algebra setting.

\section{BREUER-FredHolm THEORY}

The standard references for Breuer-Fredholm operators in a general semifinite von Neumann algebra are in Breuer [11, 12. In earlier work of some of the current authors [19] this theory was extended to handle Breuer-Fredholm operators in a skew-corner $P \mathcal{N} Q$ in the general semifinite situation with a fixed (scalar) trace $\tau$ in both the bounded and unbounded cases. All of the expected results hold but their proofs are a little more subtle. The most difficult case, index theory for unbounded Breuer-Fredholm operators will not be covered here. However, in order to handle more cases (including the case of $\tau$-finite von Neumann algebras), we allow our operators to vary within all of $\mathcal{F}^{s a}$ and not just in $\mathcal{F}_{*}^{s a}$.

If $H_{1}$ is a subspace of $H$, we denote the projection onto the closure of $H_{1}$ by $\left[H_{1}\right]$.

Definition 3.1. Let $P$ and $Q$ be projections (not necessarily infinite and not necessarily equivalent) in $\mathcal{N}$ and let $T \in P \mathcal{N} Q$. We let $\operatorname{ker}_{Q}(T)=\operatorname{ker}\left(T_{\mid Q(H)}\right)=\operatorname{ker}(T) \cap Q(H)$. The operator $T \in P \mathcal{N} Q$ is called $(P \cdot Q) \tau$-Fredholm if and only if

(1) $\left[\operatorname{ker}_{Q}(T]\right)$ and $\left[\operatorname{ker}_{P}\left(T^{*}\right)\right]$ are $\tau$-finite in $\mathcal{N}$, and

(2) there exists a projection $P_{1} \leq P$ in $\mathcal{N}$ with $P-P_{1} \tau$-finite in $\mathcal{N}$ and $P_{1}(H) \subseteq T(H)$.

In this case, we define the $(P \cdot Q)$-index of $T$ to be the number:

$$
\operatorname{Ind}_{(P \cdot Q)}(T)=\tau\left[\operatorname{ker}_{Q}(T)\right]-\tau\left[\operatorname{ker}_{P}\left(T^{*}\right)\right] .
$$

We will henceforth abbreviate this terminology to $\tau$-Fredholm or sometimes Breuer-Fredholm and drop the $(P \cdot Q)$ when there is no danger of confusion. We observe that if $P=Q$ then this 
6M-T. BENAmEUR, A. L. CAREY, J. PHILliPS, A. RENNIE, F. A. SUKOCHEV, AND K. P. WOJCIECHOWSKI

is just the definition of $\tau$-Fredholm used in Phillips et al [59] in the semifinite von Neumann algebra, $Q \mathcal{N} Q$, with the trace being the restriction of $\tau$ to $Q \mathcal{N} Q$.

We summarize the general situation of $\tau$-Fredholm operators with different domain and range [19. We re-iterate that the order of proving the usual results is crucial in developing the skewcorner case, as the various projections are neither equivalent nor infinite in general.

Lemma 3.2. Let $T \in P \mathcal{N} Q$. Then, (1) If $T$ is $(P \cdot Q)$-Fredholm, then $T^{*}$ is $(Q \cdot P)$-Fredholm and $\operatorname{Ind}\left(T^{*}\right)=-\operatorname{Ind}(T)$. If $T=V|T|$ is the polar decomposition, then $V$ is $(P \cdot Q)$-Fredholm with $\operatorname{Ind}(V)=\operatorname{Ind}(T)$ and $|T|$ is $(Q \cdot Q)$-Fredholm of index 0 .

(2) The set of all $(P \cdot Q)$-Fredholm operators in $P \mathcal{N} Q$ is open in the norm topology.

Definition 3.3. If $T \in P \mathcal{N} Q$, then a parametrix for $T$ is an operator $S \in Q \mathcal{N} P$ satisfying $S T=Q+k_{1}$ and $T S=P+k_{2}$ where $k_{1} \in \mathcal{K}_{Q \mathcal{N} Q}$ and $k_{2} \in \mathcal{K}_{P \mathcal{N} P}$.

Lemma 3.4. If the usual assumptions on $\mathcal{N}$ are satisfied, then $T \in P \mathcal{N} Q$ is $(P \cdot Q)$-Fredholm if and only if $T$ has a parametrix $S \in Q \mathcal{N} P$. Moreover, any such parametrix is $(Q \cdot P)$-Fredholm.

Proposition 3.5. Let $G, P, Q$ be projections in $\mathcal{N}$ and let $T \in P \mathcal{N} Q$ be $(P \cdot Q)$-Fredholm and $S \in G \mathcal{N} P$ be $(G \cdot P)$-Fredholm, respectively. Then, $S T$ is $(G \cdot Q)$-Fredholm and $\operatorname{Ind}(S T)=$ $\operatorname{Ind}(S)+\operatorname{Ind}(T)$.

This proof carefully adapts the original ideas of Breuer [12] in a crucial way. Finally one is easily able to deduce the following expected results.

Corollary 3.6. (Invariance properties of the $(P \cdot Q)$-Index) Let $T \in P \mathcal{N} Q$.

(1) If $T$ is $(P \cdot Q)$-Fredholm then there exists $\delta>0$ so that if $S \in P \mathcal{N} Q$ and $\|T-S\|<\delta$ then $S$ is $(P \cdot Q)$-Fredholm and $\operatorname{Ind}(S)=\operatorname{Ind}(T)$.

(2) If $T$ is $(P \cdot Q)$-Fredholm and $k \in P \mathcal{K}_{\mathcal{N}} Q$ then $T+k$ is $(P \cdot Q)$-Fredholm and $\operatorname{Ind}(T+k)=$ $\operatorname{Ind}(T)$.

\section{The ANALYTIC DEFINITION OF SPECTRAL FLOW}

4.1. Essential Codimension. If $P, Q$ are projections (not necessarily infinite) in the semifinite von Neumann algebra $\mathcal{N}$ we wish to define the essential codimension of $P$ in $Q$ whenever $\|\pi(P)-\pi(Q)\|<1$, where $\pi: \mathcal{N} \rightarrow \mathcal{Q}_{\mathcal{N}}$ is the Calkin map. Once we show that the operator $P Q \in P \mathcal{N} Q$ is a $\tau$-Fredholm operator in the sense of Section 3 then we will define the essential codimension of $P$ in $Q$ to be $\operatorname{Ind}(P Q)$. In case $\mathcal{N}=\mathcal{B}(\mathcal{H})$ a related result to the following lemma appears in Proposition 3.1 of Avron et al [5] where one of their conditions is in terms of essential spectrum. Our one condition is in terms of the norm, and the proof is very different.

Lemma 4.1. If $P, Q$ are projections in the semifinite von Neumann algebra $\mathcal{N}$ and $\pi: \mathcal{N} \rightarrow \mathcal{Q}_{\mathcal{N}}$ is the Calkin map, then $P Q \in P \mathcal{N} Q$ is $(P \cdot Q)-\tau$-Fredholm if and only if $\|\pi(P)-\pi(Q)\|<1$.

Proof. Suppose $\|\pi(Q)-\pi(P)\|<1$. Then since

$$
\|\pi(P Q P)-\pi(P)\| \leq\|\pi(Q)-\pi(P)\|<1
$$


and $\pi(P)\left(\mathcal{N} / \mathcal{K}_{\mathcal{N}}\right) \pi(P)=(P \mathcal{N} P) / \mathcal{K}_{P \mathcal{N} P}$ we see that $P Q P$ is a $\tau$-Fredholm operator in $P \mathcal{N} P$. Thus, $\operatorname{ker}_{P}(Q P) \subseteq \operatorname{ker}_{P}(P Q P)$ and so $\left[\operatorname{ker}_{P}(Q P)\right] \leq\left[\operatorname{ker}_{P}(P Q P)\right]$ where the latter is a finite projection in $P \mathcal{N} P$. Similarly, $\left[\operatorname{ker}_{Q}(P Q)\right]$ is a finite projection in $Q \mathcal{N} Q$. Since the range of $P Q$ contains the range of $P Q P$, and since this latter operator is $\tau$-Fredholm in $P \mathcal{N} P$, there is a projection $P_{1} \leq P$ so that $\tau\left(P-P_{1}\right)<\infty$ and the range of $P_{1}$ is contained in the range of $P Q$. That is, $P Q$ is $(P \cdot Q)$-Fredholm.

On the other hand, if $P Q$ is $\tau$-Fredholm then $P Q P$ is a positive $\tau$-Fredholm operator in $P \mathcal{N} P$. Letting $p=\pi(P)$ and $q=\pi(Q)$, we see that $p q p$ is an invertible positive operator in $p \mathcal{Q}_{\mathcal{N}} p$ which is $\leq p$, so $\|p-p q p\|<1$. Similarly, $\|q-q p q\|<1$. Now,

$$
(p-q)^{3}=[p-p q p]-[q-q p q]
$$

is the difference of two positive operators, so that:

$$
-[q-q p q] \leq(p-q)^{3} \leq[p-p q p] .
$$

Hence,

$$
\left\|(p-q)^{3}\right\| \leq \operatorname{Max}\{\|p-p q p\|,\|q-q p q\|\}<1 .
$$

That is,

$$
\|\pi(P)-\pi(Q)\|=\left\|(p-q)^{3}\right\|^{1 / 3}<1 .
$$

Definition 4.2. If $P$ and $Q$ are projections in $\mathcal{N}$ and if $\|\pi(P)-\pi(Q)\|<1$ then the essential codimension of $P$ in $Q$, denoted ec $(P, Q)$, is the number $\operatorname{Ind}(P Q)=\operatorname{Ind}_{(P \cdot Q)}(P Q)$. If $P \leq Q$ it is exactly the codimension of $P$ in $Q$.

Lemma 4.3. If $P_{1}, P_{2}, P_{3}$ are projections in $\mathcal{N}$ and if $\left\|\pi\left(P_{1}\right)-\pi\left(P_{2}\right)\right\|<\frac{1}{2}$ and $\| \pi\left(P_{2}\right)-$ $\pi\left(P_{3}\right) \|<\frac{1}{2}$ then ec $\left(P_{1}, P_{3}\right)=e c\left(P_{1}, P_{2}\right)+e c\left(P_{2}, P_{3}\right)$.

Proof. Since we also have $\left\|\pi\left(P_{1}\right)-\pi\left(P_{3}\right)\right\|<1$, the terms in the equation are all defined by Lemma 4.1. Translating the equation into the language of index and using Lemma 3.1 and Proposition 3.1 we see that it suffices to prove that Ind $\left(\left(P_{1} P_{3}\right)^{*}\left(P_{1} P_{2} P_{3}\right)\right)=0$. But,

$$
\begin{gathered}
\left\|\pi\left(\left(P_{1} P_{3}\right)^{*}\left(P_{1} P_{2} P_{3}\right)\right)-\pi\left(P_{3}\right)\right\|=\left\|\pi\left(P_{3} P_{1} P_{2} P_{3}\right)-\pi\left(P_{3}\right)\right\| \\
\leq\left\|\pi\left(P_{1} P_{2}\right)-\pi\left(P_{3}\right)\right\| \leq\left\|\pi\left(P_{1} P_{2}\right)-\pi\left(P_{2}\right)\right\|+\left\|\pi\left(P_{2}\right)-\pi\left(P_{3}\right)\right\| \\
\leq\left\|\pi\left(P_{1}\right)-\pi\left(P_{2}\right)\right\|+\left\|\pi\left(P_{2}\right)-\pi\left(P_{3}\right)\right\|<1 .
\end{gathered}
$$

Thus, there is a compact $k$ in $P_{3} \mathcal{N} P_{3}$ with $\left\|P_{3} P_{1} P_{2} P_{3}+k-P_{3}\right\|<1$. Hence, $\operatorname{Ind}\left(P_{3} P_{1} P_{2} P_{3}\right)=$ $\operatorname{Ind}\left(P_{3} P_{1} P_{2} P_{3}+k\right)=0$ as this latter operator is invertible in $P_{3} \mathcal{N} P_{3}$.

Remarks 4.4. If $P$ and $Q$ are projections in $\mathcal{N}$ with $\|P-Q\|<1$, then ec $(P, Q)=0$. To see this, note that $\|P Q P-P\| \leq\|Q-P\|<1$ so that $P Q P$ is invertible in $P \mathcal{N} P$ and hence range $P \supseteq$ range $P Q \supseteq$ range $P Q P=$ range $P$. Thus, range $P Q=$ range $P$ and similarly range $Q P=$ range $Q$ so the $(P \cdot Q)$ index of $P Q$ is 0 . 
8M-T. BENAMEUR, A. L. CAREY, J. PHILliPS, A. RENNIE, F. A. SUKOCHEV, AND K. P. WOJCIECHOWSKI

4.2. The general definition. Recall that $\chi=\chi_{[0, \infty)}$ is the characteristic function of the interval $[0, \infty)$ so that if $T$ is any self-adjoint operator in a von Neumann algebra $\mathcal{N}$ then $\chi(T)$ is a projection in $\mathcal{N}$.

Lemma 4.5. If $\mathcal{N}$ is a von Neumann algebra, $\mathcal{J}$ is a norm closed 2-sided ideal in $\mathcal{N}, T$ is a self-adjoint operator in $\mathcal{N}$ and $\pi(T)$ is invertible in $\mathcal{N} / \mathcal{J}$ (where $\pi: \mathcal{N} \rightarrow \mathcal{N} / \mathcal{J}$ is the quotient mapping), then $\chi(\pi(T))=\pi(\chi(T))$.

Proof. Since 0 is not in the spectrum of $\pi(T)$, the left hand side is a well-defined element of the $C^{*}$-algebra $\mathcal{N} / \mathcal{J}$. Choose $\epsilon>0$ so that $[-\epsilon, \epsilon]$ is disjoint from $\operatorname{sp}(\pi(T))$. Let $f_{1} \geq f_{2}$ be the following piecewise linear continuous functions on $\mathbf{R}$ :

$$
f_{1}(t)=\left\{\begin{array}{ll}
1 & \text { if } t \geq 0 \\
\text { linear } & \text { on }[-\epsilon, 0] \\
0 & \text { if } t \leq-\epsilon
\end{array}, \quad f_{2}(t)= \begin{cases}1 & \text { if } t \geq \epsilon \\
\text { linear } & \text { on }[0, \epsilon] . \\
0 & \text { if } t \leq 0\end{cases}\right.
$$

Now, $f_{1} \geq \chi \geq f_{2}$ on $\mathbf{R}$, but all three functions are equal on $s p(\pi(T))$. Thus,

$$
\begin{gathered}
\chi(\pi(T))=f_{1}(\pi(T))=\pi\left(f_{1}(T)\right) \geq \pi(\chi(T)) \geq \pi\left(f_{2}(T)\right) \\
=f_{2}(\pi(T))=\chi(\pi(T)) .
\end{gathered}
$$

Hence, $\chi(\pi(T))=\pi(\chi(T))$.

Definition 4.6. Let $\mathcal{N}$ be a semifinite von Neumann algebra with fixed semifinite, faithful, normal trace, $\tau$. Let $\mathcal{F}^{\text {sa }}$ denote the space of all self-adjoint $\tau$-Fredholm operators in $\mathcal{N}$. Let $\left\{B_{t}\right\}$ be any continuous path in $\mathcal{F}^{\text {sa }}$ (indexed by some interval $[a, b]$ ). Then $\left\{\chi\left(B_{t}\right)\right\}$ is a (generally discontinuous) path of projections in $\mathcal{N}$. By Lemma $4.3 \pi\left(\chi\left(B_{t}\right)\right)=\chi\left(\pi\left(B_{t}\right)\right)$ and since the spectra of $\pi\left(B_{t}\right)$ are bounded away from 0, this latter path is continuous. By compactness we can choose a partition $a=t_{0}<t_{1}<\cdots<t_{k}=b$ so that for each $i=1,2, \cdots, k$

$$
\left\|\pi\left(\chi\left(B_{t}\right)\right)-\pi\left(\chi\left(B_{s}\right)\right)\right\|<\frac{1}{2} \text { for all } t, s \text { in }\left[t_{i-1}, t_{i}\right] .
$$

Letting $P_{i}=\chi\left(B_{t_{i}}\right)$ for $i=0,1, \cdots, k$ we define the spectral flow of the path $\left\{B_{t}\right\}$ to be the number:

$$
s f\left(\left\{B_{t}\right\}\right)=\sum_{i=1}^{k} e c\left(P_{i-1}, P_{i}\right) .
$$

To see that this definition is independent of the partition, it suffices to see that it is invariant under adding a single point to the partition. However, this is exactly the content of Lemma 4.2 .

Remarks (i) If $\left\{B_{t}\right\}$ is a path in $\mathcal{F}^{s a}$ and if $t \mapsto \chi\left(B_{t}\right)$ is continuous, then $s f\left(\left\{B_{t}\right\}\right)=0$. That is, as expected heuristically, spectral flow can be nontrivial only when the path $t \mapsto \chi\left(B_{t}\right)$ has discontinuities.

(ii) For $T \in \mathcal{F}^{s a}$, let

$$
N(T)=\left\{S \in \mathcal{F}^{s a} \mid\|\pi(\chi(S))-\pi(\chi(T))\|<\frac{1}{4}\right\}
$$


Then $N(T)$ is open in $\mathcal{F}^{s a}$ since $S \mapsto \pi(\chi(S))=\chi(\pi(S))$ is continuous on $\mathcal{F}^{s a}$. Moreover, if $S_{1}, S_{2} \in N(T)$, then by the definition of spectral flow, all paths from $S_{1}$ to $S_{2}$ lying entirely in $N(T)$ have the same spectral flow, namely, ec $\left(\chi\left(S_{1}\right), \chi\left(S_{2}\right)\right)$.

Proposition 4.7. Spectral flow is homotopy invariant, that is, if $\left\{B_{t}\right\}$ and $\left\{B_{t}^{\prime}\right\}$ are two continuous paths in $\mathcal{F}^{\text {sa }}$ with $B_{0}=B_{0}^{\prime}$ and $B_{1}=B_{1}^{\prime}$ which are homotopic in $\mathcal{F}^{\text {sa }}$ via a homotopy leaving the endpoints fixed, then $s f\left(\left\{B_{t}\right\}\right)=\operatorname{sf}\left(\left\{B_{t}^{\prime}\right\}\right)$.

Proof. Let $H: I \times I \rightarrow \mathcal{F}^{s a}$ be a homotopy from $\left\{B_{t}\right\}$ to $\left\{B_{t}^{\prime}\right\}$. That is, $H$ is continuous, $H(t, 0)=B_{t}$ for all $t, H(t, 1)=B_{t}^{\prime}$ for all $t, H(0, s)=B_{0}=B_{0}^{\prime}$ for all $s$, and $H(1, s)=B_{1}=B_{1}^{\prime}$ for all $s$. By compactness we can cover the image of $H$ by a finite number of open sets $\left\{N_{1}, \cdots, N_{k}\right\}$ as in Remark 4.2. The inverse images of these open sets, $\left\{H^{-1}\left(N_{1}\right), \cdots, H^{-1}\left(N_{k}\right)\right\}$ is a finite cover of $I \times I$. Thus, there exists $\epsilon_{0}>0$ (the Lebesgue number of the cover) so that any subset of $I \times I$ of diameter $\leq \epsilon_{0}$ is contained in some element of this finite cover of $I \times I$. Thus, if we partition $I \times I$ into a grid of squares of diameter $\leq \epsilon_{0}$, then the image of each square will lie entirely within some $N_{i}$. Effectively, this breaks $H$ up into a finite sequence of "short" homotopies by restricting $H$ to $I \times J_{i}$ where $J_{i}$ are subintervals of $I$ ( of length $\leq \epsilon_{0} / \sqrt{2}$ ). These short homotopies have the added property that for fixed $J_{i}$ we can choose a single partition of $I$ so that for each subinterval $J_{\ell}$ of the partition, $H\left(J_{\ell} \times J_{i}\right)$ is contained in one of $\left\{N_{1}, \cdots, N_{k}\right\}$. By concentrating on the $i$ th "short homotopy" and relabelling $N_{1}, \cdots, N_{k}$ if necessary we can assume $H$ is such a "short homotopy." By definition, the sum of the spectral flows of the lower paths (i.e. along $I \times\{0\})$ is $s f\left(\left\{B_{t}\right\}\right)$. Since the spectral flows of the vertical paths (i.e. along $\left\{t_{k}\right\} \times J_{i}$ ) cancel in pairs, the sum of the spectral flows of the upper paths (i.e., along $\left.I \times\left\{t_{1}\right\}\right)$ equals $s f\left(\left\{B_{t}^{\prime}\right\}\right)$ and hence $s f\left(\left\{B_{t}\right\}\right)=s f\left(\left\{B_{t}^{\prime}\right\}\right)$.

Examples. If $\mathcal{N}$ is a $I I_{\infty}$ von Neumann factor with trace $\tau$ then it is well-known (and not difficult to prove) that $\mathcal{N}$ contains an abelian von Neumann subalgebra isomorphic to $L^{\infty}(\mathbf{R})$ with the property that the restriction of the trace $\tau$ to $L^{\infty}(\mathbf{R})$ coincides with the usual trace on $L^{\infty}(\mathbf{R})$ given by Lebesgue integration. We construct our first examples inside this subalgebra. Let $B_{0}$ in $L^{\infty}(\mathbf{R})$ be the continuous function:

$$
B_{0}(t)=\left\{\begin{array}{cl}
1 & \text { if } t \geq 1 \\
t & \text { if } t \in[-1,1] \\
-1 & \text { if } t \leq-1
\end{array}\right.
$$

Let $s$ be any fixed real number. Then for $t \in[0,1]$ let $B_{t}$ be defined by $B_{t}(r)=B_{0}(r+t s)$ for all $r \in \mathbf{R}$. Clearly $\left\{B_{t}\right\}$ is a continuous path in $\mathcal{F}_{*}^{s a}$. Moreover, $\chi\left(B_{t}\right)=\chi_{[-t s, \infty)}$ which differs from $\chi_{[0, \infty)}$ by the finite projection $\chi_{[-t s, 0)}$ if $s>0$ (or, $\chi_{[0,-t s)}$ if $s<0$ ). Thus, $\pi\left(\chi\left(B_{t}\right)\right)$ is constant in $\mathcal{Q}_{N}$. Hence,

$$
P_{0}=\chi\left(B_{0}\right)=\chi_{[0, \infty)}, P_{1}=\chi\left(B_{1}\right)=\chi_{[-s, \infty)}
$$

and

$$
\begin{aligned}
& s f\left(\left\{B_{t}\right\}\right)=e c\left(P_{0}, P_{1}\right)=\operatorname{Ind}\left(P_{0} P_{1}\right) \\
& =\tau\left(P_{1}-P_{0} P_{1}\right)-\tau\left(P_{0}-P_{0} P_{1}\right)=s .
\end{aligned}
$$


We note that for these examples the spectral pictures are constant! That is, $s p\left(B_{t}\right)=[-1,1]$ for all $t$ and $\operatorname{sp}\left(\pi\left(B_{t}\right)\right)=\{-1,1\}$ for all $t$. Thus, one cannot tell from the spectrum alone (even knowing the multiplicities) what the spectral flow will be.

These examples may seem paradoxical as there exists a (strong-operator topology) continuous path of unitaries $\left\{U_{t}\right\}$ so that $B_{t}=U_{t} B_{0} U_{t}^{*}$. However, there cannot exist a norm-continuous path of such unitaries as this would imply that the path $t \mapsto \chi\left(B_{t}\right)$ is a norm-continuous path of projections which it is not since $\left\|\chi\left(B_{t}\right)-\chi\left(B_{s}\right)\right\|=1$ if $s \neq t$.

On the other hand, it is not hard to prove that there is a unitary $U_{1}$ in $\mathcal{N}$ so that $B_{1}=$ $U_{1} B_{0} U_{1}^{*}$. Since the unitary group of $\mathcal{N}$ is connected in the norm topology we can find in $\mathcal{N}$ a norm continuous path $\left\{U_{t}\right\}$ of unitaries for $t \in[1,2]$ so that $U_{1}$ is as above and $U_{2}=I$. Then we can extend $\left\{B_{t}\right\}$ to a continuous loop based at $B_{0}$ by defining $B_{t}=U_{t} B_{0} U_{t}^{*}$ for $t \in[1,2]$. Since the second half of the loop satisfies $t \mapsto \chi\left(B_{t}\right)$ is norm continuous, its spectral flow is 0 and so $s f\left(\left\{B_{t}\right\}_{[1,2]}\right)=r$.

When $\mathcal{N}$ is a type $I_{\infty}$ factor we can use a similar construction with $\ell^{\infty}(\mathbf{Z})$ in place of $L^{\infty}(\mathbf{R})$ to obtain paths with any given integer as their spectral flow. Of course, these examples will not have a constant spectral picture.

Remarks 4.8. It is clear from the above definition that spectral flow does not change under reparametrization of intervals and is additive when we compose paths by concatenation. Hence, spectral flow defines a groupoid homomorphism from the homotopy groupoid, Hom $\left(\mathcal{F}_{*}^{\text {sa }}\right)$ to $\mathbf{Z}$ in the type $I_{\infty}$ factor case (respectively, to $\mathbf{R}$ in the type $I I_{\infty}$ factor case). By the examples just constructed these homomorphisms are surjective in the case of factors, even when restricted to paths based at a point $B_{0}$ in $F_{*}^{s a}$, i.e., sf : $\pi_{1}\left(\mathcal{F}_{*}^{s a}\right) \rightarrow \mathbf{Z}$ (respectively, $\mathbf{R}$ ) is surjective. To see that this group homomorphism is one-to-one on a factor requires the homotopy equivalence $\mathcal{F}_{*}^{s a} \simeq U(\infty)$ in the type $I_{\infty}$ factor case or the homotopy equivalence [55, [56] $\Omega\left(\mathcal{F}_{*}^{s a}\right) \simeq \mathcal{F}$ in the type $I I_{\infty}$ factor case: in fact, both results only need the somewhat weaker result, $\Omega\left(\mathcal{F}_{*}^{\text {sa }}\right) \simeq \mathcal{F}$.

\section{Spectral Flow Between SelF adjoint involutions}

We now revisit the special case which is naturally suggested by the definition of spectral flow. Choose projections $P, Q \in \mathcal{N}$ such that $\|\pi(P)-\pi(Q)\|<1$ so that $Q P$ is $\tau$-Fredholm. Let $B_{0}=2 Q-1, B_{1}=2 P-1$ and introduce the path $B(t)=(1-t) B_{0}+t B_{1}, 0 \leq t \leq 1$. One can easily show in this case that the path $B_{t}$ consists of Breuer-Fredholm operators. We are interested in the spectral flow along this path. By Definition 4.2 it is equal to the BreuerFredholm index of $Q P$ in $P \mathcal{N} Q$. By a careful analysis we will explain why this is the right definition.

First notice that $\operatorname{ker}_{P}(Q P)=\operatorname{ker}(Q) \cap \operatorname{ran}(P)$ and $\operatorname{ker}_{Q}(P Q)=\operatorname{ker}(P) \cap \operatorname{ran}(Q)$. A simple calculation also yields $\operatorname{ker}_{P}(Q P) \oplus \operatorname{ker}_{Q}(P Q) \subset \operatorname{ker}\left(B_{0}+B_{1}\right)$. Conversely any element $v$ of $\operatorname{ker}\left(B_{0}+B_{1}\right)$ satisfies $v=P v+Q v$ and hence $P Q(Q v)=0$ and $Q P(P v) v=0$ implying that $\operatorname{ker}\left(B_{0}+B_{1}\right) \subset \operatorname{ker}_{P}(Q P) \oplus \operatorname{ker}_{Q}(P Q)$ (note that it is elementary to check that this is an orthogonal decomposition and in particular that $\left.\operatorname{ker}_{P}(Q P) \cap \operatorname{ker}_{Q}(P Q)=\{0\}\right)$. 
Consequently to see what happens as we flow along $B(t), 0 \leq t \leq 1$ we initially track what happens in

$$
\operatorname{ker}\left(B_{0}+B_{1}\right)=\operatorname{ker}_{P}(Q P) \oplus \operatorname{ker}_{Q}(P Q) .
$$

Now for $v \in \operatorname{ker}_{P}(Q P), B_{0} v=-v, B_{1} v=v$ so that $B(t) v=(2 t-1) v$ and spectrum flows from -1 to 1 . Conversely for $v \in \operatorname{ker}_{Q}(P Q) B_{0} v=v, B_{1} v=-v$ and $B(t) v=(1-2 t) v$. Thus we get flow from 1 to -1 .

Hence the spectral flow along the path $\left\{B_{t}\right\}$, denoted $s f\left\{B_{t}\right\}$ is the index of $Q P: P H \rightarrow Q H$ as long as we can show that there cannot be spectral flow coming in some more complex way from 'outside' $\operatorname{ker}\left(B_{0}+B_{1}\right)$. We analyse this possibility below.

Remarks 5.1. Spectral flow for the path $\{B(t)\}$ actually occurs at one point, namely $t=1 / 2$. To see this we note that $B(t)$ has no kernel for $t \neq 1 / 2$ and $\operatorname{ker}(B(1 / 2))=\operatorname{ker}\left(B_{0}+B_{1}\right)$. The proof of the former assertion is elementary because if $B(t) v=0$ then $B_{0} B_{1} v=-\frac{1-t}{t} v$ so that, taking norms on both sides we deduce that $1-t \leq t$ or $t \geq 1 / 2$. Similarly $B_{1} B_{0} v=-\frac{t}{1-t} v$ so that again taking norms we obtain $t \leq 1 / 2$. Thus there is only a kernel when $t=1 / 2$.

The analysis of this example is helped by the structure of the algebra generated by $P$ and $Q$. We have:

Lemma 5.2. Let $U$ be the partial isometry in the polar decomposition of $B_{0}+B_{1}$. Then

(i) $\left(B_{0}+B_{1}\right) B(t)=B(1-t)\left(B_{0}+B_{1}\right)$

(ii) $U B(t)=B(1-t) U$ so that $U B_{0}=B_{1} U$

Proof. (i) This is a straightforward calculation.

(ii) From (i) we get $\left(B_{0}+B_{1}\right)^{2} B(t)=B(t)\left(B_{0}+B_{1}\right)^{2}$ so that

$$
U B(t)\left|B_{0}+B_{1}\right|=B(1-t) U\left|B_{0}+B_{1}\right|
$$

and hence on $\operatorname{ker}\left(B_{0}+B_{1}\right)^{\perp}$ equation (ii) of the lemma holds. Because $B(t)$ leaves the kernel of $B_{0}+B_{1}$ invariant both sides of (ii) are zero on this kernel proving the result.

In the type I factor case one can show there is always a gap in the spectrum of $B_{0}+B_{1}$ about zero. This is because on $\operatorname{ker}\left(B_{0}+B_{1}\right)^{\perp}$, the operator $B_{0}+B_{1}$ is boundedly invertible in the type I factor case so there can be no spectral flow on $\operatorname{ker}\left(B_{0}+B_{1}\right)^{\perp}$. We now show that even in a general semifinite von Neumann algebra that there can be no spectral flow when $\operatorname{ker}\left(B_{0}+B_{1}\right)=\{0\}$.

Proposition 5.3. With the above notation, if $\operatorname{ker}\left(B_{0}+B_{1}\right)=\{0\}$, then $\operatorname{sf}\{B(t)\}=0$.

Proof. By assumption

$$
\operatorname{ker}(P) \cap \operatorname{ran}(Q)=\{0\}=\operatorname{ker}(Q) \cap \operatorname{ran}(P) .
$$

Now $\mathcal{B}=\left\{1,(Q-P)^{2},(Q+P)\right\}^{\prime \prime}$ is a commutative von Neumann algebra, so that all the spectral projections of $(Q-P)^{2}$ lie in $\mathcal{B}$. Now $\left\|(Q-P)^{2}\right\|=\|Q-P\|^{2} \leq 1$ and by our assumption, 1 is not an eigenvalue of $(Q-P)^{2}$ because

$$
(Q-P)^{2} x=x \Rightarrow P(Q-P)^{2} x=P x \Rightarrow P Q P x=0 \Rightarrow(Q P)^{2} x=0
$$


18-T. BENAMEUR, A. L. CAREY, J. PHILliPS, A. RENNIE, F. A. SUKOCHEV, AND K. P. WOJCIECHOWSKI

$$
\Rightarrow(Q P) x=0 \Rightarrow P x \in \operatorname{ker}(Q) \cap \operatorname{ran}(P)=\{0\} .
$$

Hence $P x=0$ and similarly $Q x=0$. Thus $x=(Q-P)^{2} x=0$, and so $\chi_{\{1\}}\left((Q-P)^{2}\right)=0$. Now, since $\|\pi(P)-\pi(Q)\|<1$ the spectral projections

$$
p_{n}=\chi_{[1-1 / n, 1]}\left((Q-P)^{2}\right)
$$

are $\tau$-finite for large $n$, and in the commutative algebra $\mathcal{B}$. Now, the $p_{n}$ are decreasing to $\chi_{\{1\}}\left((Q-P)^{2}\right)=0$ and so $\tau\left(p_{n}\right) \rightarrow 0$. Let $\epsilon>0$ and choose $n$ so that $\tau\left(p_{n}\right)<\epsilon$, and note that

$$
p_{n}=\chi_{[-1,-\sqrt{1-1 / n}] \cup[\sqrt{1-1 / n}, 1]}((Q-P))
$$

so that $p_{n}$ commute with $Q-P$. Since $p_{n} \in \mathcal{B}$, it commutes with $Q+P$, and so commutes with both $Q$ and $P$ !

We now decompose our space with respect to $1=\left(1-p_{n}\right)+p_{n}$ and note that both $p_{n}(\mathcal{H})$ and $\left(1-p_{n}\right)(\mathcal{H})$ are left invariant by all the $B_{t}$. Hence the spectral flow will be the sum of the spectral flows on these two subspaces. Now, since $\tau\left(p_{n}\right)<\epsilon$, the maximum absolute spectral flow on $p_{n}(\mathcal{H})$ is $\left|\tau\left(p_{n}\right)\right|<\epsilon$.

On the other hand, on $\left(1-p_{n}\right)(\mathcal{H})$ we let $Q_{n}:=\left(1-p_{n}\right) Q\left(1-p_{n}\right)=Q\left(1-p_{n}\right)$ and $P_{n}:=\left(1-p_{n}\right) P\left(1-p_{n}\right)=P\left(1-p_{n}\right)$ so that

$$
B_{t}^{n}:=\left(1-p_{n}\right) B_{t}\left(1-p_{n}\right)=(1-t) Q_{n}+t P_{n} \quad \text { on }\left(1-p_{n}\right)(\mathcal{H})
$$

Now, $\left\|Q_{n}-P_{n}\right\|=\left\|\left(Q_{n}-P_{n}\right)^{2}\right\|^{1 / 2} \leq(1-1 / n)^{1 / 2}$. So $B_{0}^{n}-B_{t}^{n}=2 t\left(Q_{n}-P_{n}\right)$ and so for $t \leq 1 / 2$

$$
\left\|B_{0}^{n}-B_{t}^{n}\right\| \leq\left\|Q_{n}-P_{n}\right\| \leq(1-1 / n)^{1 / 2}=: 1-\delta_{n} .
$$

So when $t \leq 1 / 2$ we have

$$
\sigma\left(B_{t}^{n}\right) \subseteq[-1,1] \cap \overline{\operatorname{Ball}_{1-\delta_{n}}\left(\sigma\left(B_{0}\right)\right)}=\left[-1,-\delta_{n}\right] \cup\left[\delta_{n}, 1\right] .
$$

For $t>1 / 2$ we have $(1-t) \leq 1 / 2$ and $\left\|B_{1}^{n}-B_{t}^{n}\right\| \leq 1-\delta_{n}$ so again $\sigma\left(B_{t}^{n}\right) \subseteq\left[-1,-\delta_{n}\right] \cup[\delta, 1]$. Hence there can be no spectral flow on $\left(1-p_{n}\right)(\mathcal{H})$. Finally, since $\left|s f\left(B_{t}\right)\right|<\epsilon$ and $\epsilon>0$ was arbitrary, $s f\left(B_{t}\right)=0$.

5.1. The case of finite von Neumann algebras. Let us consider the case where the trace $\tau$ on $\mathcal{N}$ is finite so that for any two projections $P, Q$ in $\mathcal{N}, P-Q$ is trace class. The size of the positive part of the spectrum of $B_{0}$ is $\tau(Q)$ and the size of the positive part of the spectrum of $B_{1}$ is $\tau(P)$. Thus it is clear that $\tau(P)-\tau(Q)=\tau(P-Q)$ counts the net amount of spectrum that has moved across zero as one moves along the path $B(t), 0 \leq t \leq 1$. So the spectral flow is

$$
\tau(P-Q)=\frac{1}{2} \tau\left(B_{1}-B_{0}\right)=\frac{1}{2} \tau\left(\int_{0}^{1} \frac{d}{d t} B(t) d t\right)=\frac{1}{2} \int_{0}^{1} \tau\left(\frac{d}{d t} B(t)\right) d t .
$$

This simple observation should be compared with later formulae for spectral flow.

Now by Lemma 5.1 there is a partial isometry $U$ with $U B_{0}=B_{1} U$ on $\operatorname{ker}\left(B_{0}+B_{1}\right)^{\perp}$ so that if $R$ is the projection onto this subspace $\tau\left[R\left(B_{1}-B_{0}\right)\right]=0$. Thus as before, to calculate $\tau\left(B_{1}-B_{0}\right)$, it suffices to work in $\operatorname{ker}\left(B_{0}+B_{1}\right)=\operatorname{ker}_{Q}(P Q) \oplus \operatorname{ker}_{P}(Q P)$ and then it is clear that on this space $\tau(P-Q)=\frac{1}{2} \tau\left(B_{1}-B_{0}\right)$ is the $\tau$ dimension of $\operatorname{ker}_{P}(Q P)$ minus the $\tau$ dimension of $\operatorname{ker}_{Q}(P Q)$. 
5.2. Example: APS boundary conditions. Another way of thinking about spectral flow along $\{B(t)\}$ which is familiar from Atiyah et al [3] is to relate it to the index of the differential operator $\frac{\partial}{\partial t}+B(t)$. We will briefly sketch this connection for our example of involutions.

Let us suppose that there is a path $w(t), 0 \leq t \leq 1$ of vectors in $\mathcal{H}$ such that $w(0) \in \operatorname{ker}(Q)$ and $w(1) \in \operatorname{ran}(P)$. That is, $B_{0}(w(0))=-w(0)$ and $B_{1}(w(1))=w(1)$ so that this path represents some flow of spectrum across zero along the path $B(t), 0 \leq t \leq 1$. Assume the path is smooth and consider the equation

$$
w^{\prime}(t)+B(t) w(t)=0 .
$$

By restricting our vectors $w$ to lie in $\operatorname{ker}\left(B_{0}+B_{1}\right)$ we can easily solve this equation. First note that from

$$
\left(B_{0}+B_{1}\right) B(t)=B(1-t)\left(B_{0}+B_{1}\right)
$$

we see that $B(t)$, for each $t$ leaves $\operatorname{ker}\left(B_{0}+B_{1}\right)$ invariant. We know that for $w=w(0)$ in $\operatorname{ker}_{P}(Q P)=\operatorname{ker}(Q) \cap \operatorname{ran}(P), B(t) w=(2 t-1) w$, so (5.1) becomes $w^{\prime}(t)+(2 t-1) w(t)=0$ which has the solution:

$$
w(t)=e^{-\left(t^{2}-t\right)} w(0)
$$

noting that $w(0)=w=w(1)$ satisfies the boundary conditions. Similarly if $w \in \operatorname{ker}_{Q}(P Q)$ one easily constructs a solution to the adjoint equation

$$
-w^{\prime}(t)+B(t) w(t)=0
$$

Of course these are APS boundary conditions and we are verifying here that for the differential operator $\mathcal{B}=\frac{\partial}{\partial t}+B(t)$ with APS boundary conditions the index of $\mathcal{B}$ is the spectral flow along the path $B(t), 0 \leq t \leq 1$. More precisely $\mathcal{B}$ is densely defined on $L^{2}([0,1], \mathcal{H})$ with domain the Sobolev space of $\mathcal{H}$-valued functions on $[0,1]$ with $L^{2}$ derivative. Because $B(t)$ leaves $\operatorname{ker}\left(B_{0}+B_{1}\right)$ invariant we can solve $\mathcal{B} w=0$ separately on this space and its orthogonal complement.

Recall Lemma 5.2 where $U$, the partial isometry in the polar decomposition of $B_{0}+B_{1}$, gives an isometry from $\operatorname{ker}\left(B_{0}+B_{1}\right)^{\perp}$ to itself and satisfies $U B(t)=B(1-t) U$. Suppose then that we have $\mathcal{B} w=0$ where $w$ takes its values in $\operatorname{ker}\left(B_{0}+B_{1}\right)^{\perp}$. Then $v(t)=U w(1-t)$ satisfies the adjoint equation $\mathcal{B}^{*} v=0$ with the adjoint boundary conditions $v(0) \in Q \mathcal{H}, v(1) \in P \mathcal{H}$. In other words, each solution of $\mathcal{B} w=0$ has a counterpart solution $U w$ of the adjoint equation and vice versa. Thus, as expected, the net spectral flow on $\operatorname{ker}\left(B_{0}+B_{1}\right)^{\perp}$ must be zero.

\section{Spectral Flow FOR UNBOUNDED OPERATORS}

The framework is that of noncommutative geometry in the sense of Alain Connes [26, 27. 28, 29, 31. However we need to extend this to cover odd unbounded $\theta$-summable or finitelysummable Breuer-Fredholm modules for a unital Banach $*$-algebra, $\mathcal{A}$. These are pairs $(\mathcal{N}, D)$ where $\mathcal{A}$ is represented in the semifinite von Neumann algebra $\mathcal{N}$ with fixed faithful, normal semifinite trace $\tau$ acting on a Hilbert space, $H$, and $D$ is an unbounded self-adjoint operator on $H$ affiliated with $\mathcal{N}$ satisfying: $\left(1+D^{2}\right)^{-1}$ is compact with the additional side condition that either $e^{-t D^{2}}$ is trace class for all $t>0(\theta$-summable $)$ or $\left(1+D^{2}\right)^{-1 / 2} \in \mathcal{L}^{n}$ for all $n>p$ 
(with $p$ chosen to be the least real number for which this holds) and $[D, a]$ is bounded for all $a$ in a dense $*$-subalgebra of $\mathcal{A}$. The condition $\left(1+D^{2}\right)^{-1 / 2} \in \mathcal{L}^{n}$ is known as $n$-summability. An alternative terminology is to refer to $(\mathcal{A}, \mathcal{N}, D)$ as a semifinite spectral triple. The theory of spectral triples in a von Neuman algebra was first exposed in Carey et al [15] and further developed by Benameur et al [6] and some of the present authors [20, 17, 18, 19, 66].

If $u$ is a unitary in this dense $*$-subalgebra then

$$
u D u^{*}=D+u\left[D, u^{*}\right]=D+B
$$

where $B$ is a bounded self-adjoint operator in $\mathcal{N}$. We say $D$ and $u D u^{*}$ are gauge equivalent. The path

$$
D_{t}^{u}:=(1-t) D+t u D u^{*}=D+t B
$$

is a "continuous" path of unbounded self-adjoint Breuer-Fredholm operators. More precisely,

$$
F_{t}^{u}:=D_{t}^{u}\left(1+\left(D_{t}^{u}\right)^{2}\right)^{-1 / 2}
$$

is a norm-continuous path of (bounded) self-adjoint $\tau$-Breuer-Fredholm operators. The spectral flow along this path $\left\{F_{t}^{u}\right\}$ (or $\left\{D_{t}^{u}\right\}$ ) is defined using the first Section via $s f\left(\left\{D_{t}^{u}\right\}\right):=s f\left(\left\{F_{t}^{u}\right\}\right)$. It recovers the pairing of the K-homology class $[D]$ with the K-theory class $[u]$.

We can relate this spectral flow for the path $\left\{D_{t}^{u}\right\}$ of unbounded Breuer Fredholm operators to the relative index of two projections as follows. Let $\tilde{F}_{0}^{u}$ and $\tilde{F}_{1}^{u}$ be the partial isometries in the polar decomposition of $F_{0}^{u}$ and $F_{1}^{u}$ respectively. By convention these extend to unitaries by making them the identity on $\operatorname{ker}\left(F_{0}^{u}\right)$ and $\operatorname{ker}\left(F_{1}^{u}\right)$ respectively. We introduce the path $\left\{\tilde{F}_{t}^{u}\right\}$ where $\tilde{F}_{t}^{u}=(1-t) \tilde{F}_{0}^{u}+t \tilde{F}_{1}^{u}$. We show below that the spectral flow along $\left\{F_{t}^{u}\right\}$ is in fact equal to the spectral flow along $\left\{\tilde{F}_{t}^{u}\right\}$.

Proposition 6.1. Let $(\mathcal{A}, \mathcal{N}, D)$ be a semifinite spectral triple, and let $u \in \mathcal{A}$ be a unitary. Then the spectral flow from $D$ to $u D u^{*}$ is $\operatorname{sf}\left(D, u D u^{*}\right)=\operatorname{Ind}(P u P)$, where $P:=\chi(D)$.

Proof. By the definition $s f\left(D, u D u^{*}\right):=s f\left(F_{D}, F_{u D u^{*}}\right)$, where $F_{D}:=D\left(1+D^{2}\right)^{-1 / 2}$. With $\tilde{F}_{D}$ as defined in the paragraph preceding the proposition introduce the non-negative spectral projection $P$ of $F_{D}$ by $\tilde{F}_{D}=2 P-1, \tilde{F}_{u D u^{*}}=2 Q-1=2\left(u P u^{*}\right)-1$. If $\|\pi(P)-\pi(Q)\|<1$, then by the definition

$$
s f\left(F_{D}, F_{u D u^{*}}\right):=\operatorname{Ind}(P Q)=e c(P, Q) .
$$

To see that $\|\pi(P)-\pi(Q)\|<1$, we have [15], $F_{D}-F_{u D u^{*}} \in \mathcal{K}_{\tau \mathcal{N}}$ and

$$
\begin{aligned}
\tilde{F}_{D}-F_{D} & =\tilde{F}_{D}\left(1-\left|F_{D}\right|\right)=\tilde{F}_{D}\left(1-\left|F_{D}\right|^{2}\right)\left(1+\left|F_{D}\right|\right)^{-1} \\
& =\tilde{F}_{D}\left(1+D^{2}\right)^{-1}\left(1+\left|F_{D}\right|\right)^{-1} \in \mathcal{K}_{\tau \mathcal{N}} .
\end{aligned}
$$

Hence,

$$
2(P-Q)=\tilde{F}_{D}-\tilde{F}_{u D u^{*}}=\left(\tilde{F}_{D}-F_{D}\right)+\left(F_{D}-F_{u D u^{*}}\right)+\left(F_{u D u^{*}}-\tilde{F}_{u D u^{*}}\right)
$$

is also $\tau$-compact, and therefore $\|\pi(P)-\pi(Q)\|=0<1$. By Lemma 4 .1 this shows that the operator $P Q$ is $(P \cdot Q)$-Fredholm. Hence, using the formula $\operatorname{Ind}(S T)=\operatorname{Ind}(S)+\operatorname{Ind}(T)$ from Section 3 above, we obtain

$$
s f\left(D, u D u^{*}\right)=\operatorname{Ind}(P Q)=\operatorname{Ind}\left(P u P u^{*}\right)=\operatorname{Ind}(P u P) .
$$


We conclude this Section with a discussion of a theorem of Lesch [46]. Let $\mathcal{A}$ be a unital $C^{*}$ algebra with a faithful tracial state $\tau,\left(\pi_{\tau}, \mathcal{H}_{\tau}\right)$ be the GNS representation of $\mathcal{A}$. Let $(\mathcal{A}, \mathbf{R}, \alpha)$ be a $\tau$-invariant $C^{*}$-dynamical system. We will identify $\mathcal{A}$ with its image $\pi_{\tau}(\mathcal{A})$. Let $\mathcal{A} \times{ }_{\alpha} \mathbf{R}$ be the crossed product, so it acts on $\mathcal{H}=L^{2}\left(\mathbf{R}, \mathcal{H}_{\tau}\right)=L^{2}(\mathbf{R}) \otimes \mathcal{H}_{\tau}$. So we have representations $\pi$ and $\lambda$ of $\mathcal{A}$ and $\mathbf{R}$ given as follows: $\pi(a)$ acts on $\xi \in \mathcal{H}$ by $\pi(a) \xi(s)=\alpha_{s}^{-1}(a) \xi(s)$ and $\lambda_{t} \xi(s)=\xi(s-t)$. Let $\mathcal{N}$ be the von Neumann algebra generated by $\mathcal{A} \times{ }_{\alpha} \mathbf{R}$. Clearly, $\lambda=\left\{\lambda_{t}\right\}_{t \in \mathbf{R}}$ is a oneparameter group of unitaries in $\mathcal{N}$. Let $D$ be its infinitesimal generator, that is $\lambda_{t}=e^{-i t D}, t \in$ $\mathbf{R}$. We have $\pi\left(\alpha_{t}(a)\right)=\lambda_{t} \pi(a) \lambda_{-t}$, which is equivalent to $\pi(\delta(a))=2 \pi i[D, \pi(a)]$, where $\delta$ is the infinitesimal generator of $\alpha_{t}$ and $a$ is in the domain of $\delta$ which is a dense ${ }^{*}$-subalgebra $\mathcal{A}_{0}$ of $\mathcal{A}$.

In this situation a combination of Proposition 6.1] and earlier work [21] gives the following index theorem of Lesch [46] and Phillips and Raeburn [59].

Theorem 6.2. The triple $\left(\mathcal{A}_{0}, \mathcal{H}, D\right)$ is a $(1, \infty)$-summable semifinite spectral triple and for

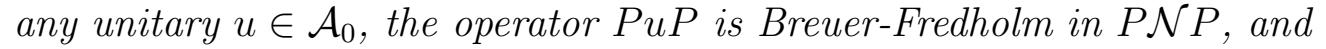

$$
s f\left(D, u D u^{*}\right)=\operatorname{Ind}(P u P)=\frac{1}{2 \pi i} \tau\left(u \delta\left(u^{*}\right)\right),
$$

where $P=\chi_{[0, \infty)}(D)$.

In the case when $\mathcal{A}=C(\mathbf{T}), \alpha_{t}$ is the rotation by an angle $t$ and $\tau$ is the arclength integral on $\mathbf{T}$, then modulo some fiddling with $\mathbf{R}$ vs. $\mathbf{T}$, we infer the classical Gohberg-Krein theorem [1].

Corollary 6.3. If $u$ is a unitary in $C(\mathbf{T})$ which is continuously differentiable then

$$
s f\left(D, u D u^{*}\right)=\operatorname{Ind}(P u P)=\frac{-1}{2 \pi i} \int_{\mathbf{T}} \frac{u^{\prime}(x)}{u(x)} d x .
$$

In the case when $\mathcal{A}=C A P(\mathbf{R})=C\left(\mathbf{R}_{B}\right)$ (i.e. $\mathcal{A}$ is the $C^{*}$-algebra of all uniformly almost periodic functions on $\mathbf{R}$, which we identify with the $C^{*}$-algebra of all continuous functions on the Bohr compactification $\mathbf{R}_{B}$ ), $\alpha_{t}$ is a shift by $t$ and $\tau$ is the Haar integral on $\mathbf{R}_{B}$, we immediately infer the Coburn-Douglas-Schaeffer-Singer theorem.

Corollary 6.4. If $u$ is a unitary almost periodic continuously differentiable function then

$$
s f\left(D, u D u^{*}\right)=\operatorname{Ind}(P u P)=\lim _{T \rightarrow \infty} \frac{-1}{4 \pi i T} \int_{-T}^{T} \frac{u^{\prime}(x)}{u(x)} d x .
$$

In the case when $\mathcal{A}=C\left(\mathbf{T}^{2}\right), \alpha_{t}$ is the Kronecker flow given by the vector field $\partial_{x}+\theta \partial_{y}$ with an irrational angle $\theta$ and $\tau$ is the Haar integral on $\mathbf{T}^{2}$ we get the following example [15].

Corollary 6.5. For the unitary element $u\left(z_{1}, z_{2}\right)=z_{2}$ from $C\left(\mathbf{T}^{2}\right)$, we have $s f\left(D, u D u^{*}\right)=$ $\operatorname{Ind}(P u P)=\theta$. 


\section{Fredholm modules AND FORMUlae FOR SPECTRAL FLOW}

In the case of a finite von Neumann algebra with finite trace $\tau$ for any projections $P$ and $Q, P-Q$ is trivially trace class. For a general semi-finite von Neumann algebra $\mathcal{N}$ arbitrary projections do not satisfy this property. However summability conditions on $D$ guarantee that there is a function $f$ such that $f(P-Q)$ is trace class. In this setting Carey and Phillips [16] extended results of Avron et al [5. Specifically, provided $f(1)$ is nonvanishing and $f$ is odd with $f(P-Q)$ trace class, then

$$
s f\{B(t)\}=\operatorname{Ind} Q P=\frac{1}{f(1)} \tau(f(P-Q)) .
$$

Starting from these results a somewhat lengthy argument produces general formulae for spectral flow in the case of $p$-summable and $\Theta$-summable unbounded Fredholm modules [15, 16] which we will now describe.

7.1. The Carey-Phillips Formulae For Spectral Flow. It was Singer 65 who suggested, in the 1974 Vancouver ICM, that spectral flow and eta invariants were given by integrating a one form. The first paper to systematically exploit this observation was that of Getzler [40]. Getzler's paper provided the inspiration for the following extensions. For paths of Dirac type operators formulae analogous to the ones we describe here go back to the original papers [3], see for example Chapter 8 of Melrose [52].

Let $(\mathcal{A}, \mathcal{H}, D)$ be a $\theta$-summable spectral triple. We focus here on a couple of the main results of Carey et al [16] in the particular case where we compute the spectral flow from $D_{0}$ to $D_{1}=u D_{0} u^{*}$. First we have the formula

$$
s f\left(D, u D u^{*}\right)=\frac{1}{\sqrt{\pi}} \int_{0}^{1} \tau\left(u\left[D, u^{*}\right] e^{-\left(D+t u\left[D, u^{*}\right]\right)^{2}}\right) d t .
$$

If $(\mathcal{A}, \mathcal{H}, D)$ is $n$-summable for some $n>1$ then

$$
s f\left(D, u D u^{*}\right)=\frac{1}{C_{n / 2}} \int_{0}^{1} \tau\left(u\left[D, u^{*}\right]\left(1+\left(D+t u\left[D, u^{*}\right]\right)^{2}\right)^{-n / 2}\right) d t,
$$

with $C_{n / 2}=\int_{-\infty}^{\infty}\left(1+x^{2}\right)^{-n / 2} d x$.

In the type I case the theta summable formula appeared in Geztler [40. The proof of this formula in general uses a result on spectral flow for bounded self adjoint Breuer-Fredholm operators which we will briefly explain.

7.2. Paths of unbounded Breuer-Fredholm operators. Our approach to spectral flow for a path of unbounded self adjoint operators affiliated to $\mathcal{N}$ is to introduce the map $D \mapsto F_{D}=$ $D\left(1+D^{2}\right)^{-1 / 2}$. By Carey et al [19], Section 3 if $\{D(t)\}=\{D(0)+A(t)\}$ is a path of unbounded self adjoint $\tau$-Breuer-Fredholm operators affiliated to $\mathcal{N}$ where $\{A(s)\}$ is a norm continuous path of bounded self adjoint operators in $\mathcal{N}$, then $\left\{F_{D(t)}\right\}$ is a continuous path of self adjoint $\tau$-Breuer-Fredholm operators in $\mathcal{N}$. We then define the spectral flow of the path $\{D(t)\}$ to be the spectral flow of the path $\left\{F_{D(t)}\right\}$, and note that in the case $\mathcal{N}=\mathcal{B}(\mathcal{H})$, by Booß-Bavnbek et al [9] one can define $s f(\{D(s)\})$ directly. 
The principal difficulty introduced by this point of view is that in practice the map $D(t) \mapsto$ $F_{D(t)}$ is hard to deal with when it comes to proving continuity and differentiability. One of the main features of earlier work [16] was to surmount this hurdle.

It is easier to deal with the map $s \mapsto(\lambda-D(t))^{-1}$ where $\lambda$ is in the resolvent set of $D(t)$ and to require continuity of this map into the bounded operators in $\mathcal{N}$. This is equivalent to graph norm continuity. It is shown that [9, 47] for $\mathcal{N}=\mathcal{B}(\mathcal{H})$ this resolvent map suffices for a definition of spectral flow for paths of unbounded self adjoint Fredholm operators. Unfortunately the case of general semifinite $\mathcal{N}$ seems beyond the scope of these methods.

We let $\mathcal{M}_{0}=\left\{D=D_{0}+A \mid A \in \mathcal{N}_{s a}\right\}$ be an affine space modelled on $\mathcal{N}_{s a}$. Let $\gamma=\left\{D_{t}=\right.$ $\left.D_{0}+A(t), a \leq t \leq b\right\}$ be a piecewise $C^{1}$ path in $\mathcal{M}_{0}$ with $D_{a}$ and $D_{b}$ invertible. The spectral flow formula of Getzler [40] when $\mathcal{N}=B(H)$ is

$$
\begin{aligned}
\operatorname{sf}\left(D_{a}, D_{b}\right) & =-\int_{\gamma} \alpha_{\epsilon}+\frac{1}{2} \eta_{\epsilon}\left(D_{b}\right)-\frac{1}{2} \eta_{\epsilon}\left(D_{a}\right) \\
& =\sqrt{\frac{\epsilon}{\pi}} \int_{a}^{b} \tau\left(\frac{d}{d t}\left(D_{t}\right) e^{-\epsilon D_{t}^{2}}\right) d t+\frac{1}{2} \eta_{\epsilon}\left(D_{b}\right)-\frac{1}{2} \eta_{\epsilon}\left(D_{a}\right) .
\end{aligned}
$$

where $\eta_{\epsilon}(D)$ are approximate eta invariant correction terms:

$$
\eta_{\epsilon}(D)=\frac{1}{\sqrt{\pi}} \int_{\epsilon}^{\infty} \tau\left(D e^{-t D^{2}}\right) t^{-1 / 2} d t
$$

and $\alpha_{\epsilon}$ is a one form defined on $\mathcal{N}_{s a}$, the tangent space to $\mathcal{M}_{0}$, via $\alpha_{\epsilon}(X)=\sqrt{\frac{\epsilon}{\pi}} \tau\left(X e^{-\epsilon D^{2}}\right)$.

The most general formula in the bounded case deals with a pair of self-adjoint $\tau$-BreuerFredholm operators $\left\{F_{j}, j=1,2\right\}$, joined by a piecewise $C^{1}$ path $\left\{F_{t}\right\}, t \in[1,2]$ in a certain affine subspace of the space of all self-adjoint $\tau$-Breuer-Fredholm operators. The spectral flow along such a path is given by

$$
s f\left(F_{1}, F_{2}\right)=\frac{1}{C} \int_{1}^{2} \tau\left(\frac{d}{d t}\left(F_{t}\right)\left|1-F_{t}^{2}\right|^{-r} e^{-\left|1-F_{t}^{2}\right|^{-\sigma}}\right) d t+\gamma\left(F_{2}\right)-\gamma\left(F_{1}\right)
$$

where the $\gamma\left(F_{j}\right)$ are eta invariant type correction terms, $C$ is a normalization constant depending on the parameters $r \geq 0$ and $\sigma \geq 1$. The affine space in which $\left\{F_{t}\right\}$ live is defined in terms of perturbations of one fixed $F_{0}$ and by the condition that $\left|1-F_{t}^{2}\right|^{-r} e^{-\left|1-F_{t}^{2}\right|^{-\sigma}}$ is trace class [16. To see one important place where such complicated formulae arise, one takes the Getzler expression with $\epsilon=1$ and where the endpoints are unitarily equivalent so that the end-point correction terms cancel:

$$
\frac{1}{\sqrt{\pi}} \int_{a}^{b} \tau\left(\frac{d}{d t}\left(D_{t}\right) e^{-D_{t}^{2}}\right) d t
$$

and does the change of variable $F_{t}=D_{t}\left(1+D_{t}^{2}\right)^{-1 / 2}$. Then, $\left(1+D_{t}^{2}\right)^{-1}=\left(1-F_{t}^{2}\right)=\left|1-F_{t}^{2}\right|$, and if one is careless and just differentiates formally (not worrying about the order of the factors), one obtains the expression:

$$
\frac{e}{\sqrt{\pi}} \int_{a}^{b} \tau\left(\frac{d}{d t}\left(F_{t}\right)\left|1-F_{t}^{2}\right|^{-3 / 2} e^{\left|1-F_{t}^{2}\right|^{-1}}\right) d t
$$


While the actual details are much more complicated, this is the heuristic essence of the reduction of the unbounded case to the bounded case.

The key observation in this approach is a geometric viewpoint due to Getzler. He noted [40] that in the unbounded case the integrand in the spectral flow formula is a one form on the affine space $\mathcal{M}_{0}$. This goes back to the observation by Singer that the eta invariant itself is actually a one form. One may also explain this fact from our point of view [16]. In proving the bounded spectral flow formula one uses in a crucial way the fact that $D \rightarrow \alpha_{D}$ where $\alpha_{D}(X)=\tau\left(X e^{\left|1-F_{D}^{2}\right|^{-1}}\right)$ for $X$ in the tangent space to $\mathcal{M}_{0}$ at $D$ is an exact one form.

Example. We revisit Corollary 6.3. The straight line path from $D$ to $u D u^{*}$ is $D_{t}^{u}=D+t \theta 1$ for $t \in[0,1]$. As $t$ increases from 0 to 1 , the spectral subspaces of the operators $D_{t}^{u}$ remain the same, but the spectral values each increase by $\theta$. The spectral subspace of $D$ corresponding to the interval $[-\theta, 0), E=E_{[-\theta, 0)}$, is exactly the subspace where the spectral values change from negative to non-negative. By a calculation very similar to the example from Section 4.2, the spectral flow of the path $\left\{D_{t}^{u}\right\}$ is exactly $\tau(E)$ and since $E=\lambda(\hat{g}) \otimes 1$ where $g=\chi_{[-\theta, 0)}$ we have

$$
s f\left(\left\{D_{t}^{u}\right\}\right)=\tau(E)=\int_{-\infty}^{\infty} \chi_{[-\theta, 0)} d r=\theta .
$$

It is also easy to verify directly that $\theta$ is the Breuer-Fredholm index of the operator $T_{u}:=P u P$ in the $\mathrm{II}_{\infty}$ factor $P \mathcal{N} P$. Finally, using the formula of Section 7.1 with $n / 2=1$ we calculate:

$$
\begin{array}{r}
\frac{1}{\pi} \int_{0}^{1} \tau\left(\frac{d}{d t}\left(D_{t}^{u}\right)\left(1+\left(D_{t}^{u}\right)^{2}\right)^{-1}\right) d t=\frac{1}{\pi} \int_{0}^{1} \tau\left(\theta\left(1+(D+t \theta)^{2}\right)^{-1}\right) d t \\
=\frac{\theta}{\pi} \int_{0}^{1}\left(\int_{-\infty}^{\infty} \frac{1}{1+(r+t \theta)^{2}} d r\right) d t=\frac{\theta}{\pi} \int_{0}^{1}\left(\int_{-\infty}^{\infty} \frac{1}{1+u^{2}} d u\right) d t=\theta
\end{array}
$$

which gives the expected result $\operatorname{Ind}(P u P)=s f\left(\left\{D_{t}^{u}\right\}\right)=\theta$.

\section{Spectral Flow, Adiabatic Limits and Covering Spaces}

8.1. Introductory Remarks. We start with some observations of Mathai [49, 48, on the motivating example which arises from the fundamental paper of Atiyah [1. Assume that $D_{0}$ is a self adjoint Dirac type operator on smooth sections of a bundle over an odd dimensional manifold $M$. We assume that $M$ is not compact but admits a continuous free action of a discrete group $G$ such that the quotient of $M$ by $G$ is a compact manifold. We assume $M$ is equipped with a $G$ invariant metric in terms of which $D_{0}$ is defined and from which we obtain an Hermitian inner product on the sections of the bundle such that $D_{0}$ is unbounded and self-adjoint on an appropriate domain. In this setting the $G$ action on $M$ lifts to an action by unitary operators on $L^{2}$ sections of the bundle. The von Neumann algebra $\mathcal{N}$ we consider is the commutant of the $G$ action on $L^{2}$ sections [49]. Consider a path of the form $D_{t}=D_{0}+A(t)$ where $A(t)$ is a bounded self adjoint pseudodifferential operator depending continuously on $t$ in 
the norm topology and commuting with the $G$ action. Then $D_{t}$ is self adjoint Breuer-Fredholm operator affiliated to $\mathcal{N}$, and $D_{t}\left(1+D_{t}^{2}\right)^{-1 / 2}$ is a bounded self adjoint Breuer-Fredholm operator in $\mathcal{N}$ for each $t \in \mathbf{R}$.

While $\mathcal{N}$ is a semifinite von Neumann algebra it is not in general a factor. There is a natural trace on $\mathcal{N}$ (considered by Atiyah [1] in his account of the $L^{2}$ index theorem) which we now define. It will be with respect to this trace that we calculate spectral flow along $\left\{D_{0}+A(t)\right\}$ (recall that the type II spectral flow depends on the choice of trace non-trivially when the algebra $\mathcal{N}$ has non-trival centre). On operators with smooth Schwartz kernel $k(x, y) ; x, y \in M$ the trace $\tau_{G}$ is given by taking the fibrewise trace of the kernel on the diagonal $\operatorname{tr}(k(x, x))$ and integrating over a fundamental domain for $G$. This is the natural trace as may be seen by recognising that the representation of $G$ we obtain here is quasi-equivalent to the regular representation. The regular representation is determined by the standard trace $\tau_{0}$ which is given on an element $\sum \lambda_{g} g$ of the group algebra by $\tau_{0}\left(\sum \lambda_{g} g\right)=\lambda_{e}$ (with the identity being $e \in G$ and the $\left.\lambda_{g} \in \mathbf{C}\right)$.

The analysis of spectral flow traditionally proceeds by replacing $M$ by $M \times S^{1}$ or $M \times[0,1]$ and considering the Dirac operator on this even dimensional manifold as in Mathai [49]. On covering spaces it is believed by the experts that one should be able to recover an analytic spectral flow formula however one cannot easily extract a proof from the literature. The argument we present in this Section shows how a special case of the spectral flow formulae discussed in the previous Section arises naturally from adiabatic limit ideas due originally to Cheeger [22].

8.2. Easy Adiabatic Formula in even dimensions (EAF). We use an adiabatic process, which leads to the formula for the leading term in the expansion of the (difference of) heat kernels. This is part of an IUPUI preprint [67] which was never submitted for publication. We discuss the simplest possible case (of a compatible Dirac operator with coefficients in an auxiliary bundle) in order to avoid use of elliptic estimates as was done in Cheeger 22]. Therefore our main tool is Duhamel's principle (the expansion of the heat kernels of the Dirac Laplacians with respect to the perturbation terms [10, 51]) and we call our result the EAF = Easy Adiabatic Formula. (Let us point out that it is not difficult to imitate Cheeger's proof and obtain the $E A F$ in complete generality i.e. for the family of Dirac operators with varying first order part.) We present a proof in the case of a closed manifold $M$ and later on outline why our argument holds in the case of a continuous free action of a discrete group.

Let $B: C^{\infty}(M ; S) \rightarrow C^{\infty}(M ; S)$ denote a compatible Dirac operator acting on sections of a bundle of Clifford modules $S$ over a closed, odd-dimensional manifold $M$ [10]. Introduce an auxiliary hermitian vector bundle $E$ with hermitian connection $\nabla$, and the operator $B_{0}=$ $B \otimes_{\nabla} I d_{E}$ (see Palais [53], Chapter IV). Let $g: E \rightarrow E$ denote a unitary bundle automorphism, then we can introduce the operator

$$
B_{1}=g B_{0} g^{-1}=(I d \otimes g)\left(B \otimes \nabla I d_{E}\right)\left(I d \otimes g^{-1}\right) .
$$

The difference $T=B_{1}-B_{0}=[g, B] g^{-1}$ is a bundle endomorphism and we want to present a formula for the spectral flow of the family

$$
\left\{B_{u}=B_{0}+u T\right\}_{0 \leq u \leq 1} .
$$


Spectral flow is a homotopy invariant, so we can restrict ourselves to the study of the spectral flow of a smooth family of self-adjoint operators over $S^{1}$. We introduce a smooth cut-off function $\alpha: \mathbf{R} \rightarrow \mathbf{R}$, such that

$$
\alpha(u)=\left\{\begin{array}{c}
0 \quad \text { if } \quad u \leq 1 / 4, \\
1 \quad \text { if } 3 / 4 \leq u .
\end{array}\right.
$$

We may also assume that there exists a positive constant $c$, such that

$$
\left|\frac{d^{k} \alpha}{d u^{k}}\right| \leq c \cdot u
$$

for $0 \leq u \leq 1, k=0,1,2$. Now, we consider the family

$$
\left\{B_{u}=B_{0}+\alpha(u) T\right\},
$$

which in an obvious way provides us with a family of operators on $S^{1}$. We may also consider the corresponding operator $\mathcal{D}=\partial_{u}+B_{u}$ on the closed manifold $N=S^{1} \times M$ where $B_{u}$ is given by the formula (8.3) . The operator $\mathcal{D}$ acts on sections of the bundle $[0,1] \times S \otimes E / \cong$, where the identification is given by

$$
(1, y ; g(y) w) \cong(0, y ; w) \text { where } w \in S_{y} \otimes E_{y} .
$$

Theorem 8.1. The following formula holds for any $t>0$

$$
\text { index } \mathcal{D}=s f\left\{B_{u}\right\}=\sqrt{\frac{t}{\pi}} \cdot \int_{0}^{1} \operatorname{Tr}_{M} \dot{B}_{u} e^{-t B_{u}^{2}} d u
$$

where as usual $\dot{B}_{u}=\frac{d B}{d u}$.

The first equality in (8.4) goes back to the original Atiyah-Patodi-Singer paper [3]. They also proved a formula

$$
s f\left\{B_{u}\right\}=\int_{0}^{1} \dot{\eta}_{u} d u,
$$

where $\eta_{u}$ denotes the $\eta$-invariant of the operator $B_{u}$. The equality

$$
s f\left\{B_{u}\right\}=\sqrt{\frac{t}{\pi}} \cdot \int_{0}^{1} \operatorname{Tr}_{M} \dot{B}_{u} e^{-t B_{u}^{2}} d u
$$

and more (see below) was proved by the last named author around 1991 and published in one of his IUPUI preprints 67]. The formal paper, which was supposed to contain a new discussion of Witten's Holonomy Theorem never appeared and the result eventually resurfaced in the paper by Getzler [40. We have to mention that it is not difficult to manufacture a more straightforward argument to prove the second equality in (8.4). Here we prove a stronger result that provides the "adiabatic" equality on the level of heat kernels, from which (8.4) and other results not covered in the current paper follow. The original proof was a "toy" model for a simplified version of Cheeger's proof of Witten's Holonomy Theorem [22] and proves the corresponding adiabatic equality on the level of the kernels of the corresponding heat operators. Therefore we call this equality the $E A F=$ Easy Adiabatic Formula. 
The $E A F$ is obtained by applying the adiabatic process on $N$ in the normal direction to a fibre $M$. We replace the product Riemannian metric $g=d u^{2}+g_{M}$ by a new metric

$$
g_{\epsilon}=\frac{d u^{2}}{\epsilon^{2}}+g_{M}=d v^{2}+g_{M},
$$

and let the positive parameter $\epsilon$ run to 0 . The corresponding operator $\mathcal{D}_{\epsilon}$ has the following representation

$$
\mathcal{D}_{\epsilon}(v, y)=\partial_{v}+B_{\epsilon v}(y)
$$

In the equality (8.6) we use a new normal coordinate $v=\frac{u}{\epsilon}(y \in M)$. The operator $\mathcal{D}_{\epsilon}$ lives on the manifold $N_{\epsilon}=S_{\epsilon}^{1} \times M$, where $S_{\epsilon}^{1}$ denotes the circle of length $\frac{1}{\epsilon}$. Both index and $s f$ do not change their values under the deformation, hence we have the equality

$$
\text { index } \mathcal{D}=\operatorname{index} \mathcal{D}_{\epsilon}=\operatorname{sf}\left\{B_{\epsilon v}\right\}=\operatorname{sf}\left\{B_{u}\right\} .
$$

The famous McKean-Singer equality expresses the index in terms of the kernels of the heat operators

$$
\text { index } \mathcal{D}=\operatorname{index} \mathcal{D}_{\epsilon}=\operatorname{Tr} e^{-t \mathcal{D}_{\epsilon}^{*} \mathcal{D}_{\epsilon}}-\operatorname{Tr} e^{-t \mathcal{D}_{\epsilon} \mathcal{D}_{\epsilon}^{*}}
$$

for fixed $t>0$ as $\epsilon \rightarrow 0$. Let $k_{\epsilon}\left(t ;\left(v_{1}, y_{1}\right),\left(v_{2}, y_{2}\right)\right)\left(v_{i}\right.$ is the coordinate on $S_{\epsilon}^{1}$ and $\left.y_{i} \in M\right)$, denote the kernel of the operator $e^{-t \mathcal{D}^{*} \mathcal{D}_{\epsilon}}-e^{-t \mathcal{D}_{\epsilon} \mathcal{D}_{\epsilon}^{*}}$. This is the difference of the heat kernels, which are pointwise bounded for fixed time $t$ (see Proposition 8.5), which expands into expansion with respect to the parameter $\epsilon$. The contributions to the leading term from the kernel of the operator $\mathcal{D}^{*} \mathcal{D}$ and the kernel of the operator $\mathcal{D D}^{*}$ cancel each other, hence this term is equal to 0 . Theorem 8.2 provides the formula for the second term in the expansion. It follows that the kernel $k_{\epsilon}\left(t ;\left(v_{1}, y_{1}\right),\left(v_{2}, y_{2}\right)\right)$ at the given point is of size of $\epsilon$ and the volume of the manifold $N_{\epsilon}$ is equal to $\frac{1}{\epsilon} \cdot \operatorname{vol}(M)$ hence at the end we obtain a finite limit

$$
\lim _{\epsilon \rightarrow 0} \int_{N_{\epsilon}} \operatorname{tr} k_{\epsilon}(t ;(v, y),(v, y)) d v d y
$$

equal to (8.4).

Now, we present the kernel on $N_{\epsilon}$, which gives the leading term in the expansion of $k_{\epsilon}\left(t ;\left(v_{1}, y_{1}\right),\left(v_{2}, y_{2}\right)\right)$. Let us fix $v_{0}$ the value of the normal coordinate and let $e_{v_{0}}\left(t ;\left(v_{1}, y_{1}\right),\left(v_{2}, y_{2}\right)\right)$ denote the kernel of the heat operator $e^{-t B_{\epsilon v_{0}}^{2}}$. We also introduce $\epsilon \alpha^{\prime}\left(\epsilon v_{0}\right) T e_{v_{0}}$, kernel of the operator $\epsilon \alpha^{\prime}\left(\epsilon v_{0}\right) T e^{-t B_{\epsilon v_{0}}^{2}}$. To get the final product we have to take the convolution of kernels. If $k_{1}, k_{2}$ denote two time-dependent operators with smooth kernels on $M$, then $k_{1} * k_{2}(t)=$ $\int_{0}^{t} k_{1}(s) k_{2}(t-s) d s$ and on the level of the kernels we have the equality

$$
k_{1} * k_{2}\left(t ; y_{1}, y_{2}\right)=\int_{0}^{t} d s \int_{M} d z k_{1}\left(s ; y_{1}, z\right) k_{2}\left(t-s ; z, y_{2}\right)
$$

We introduce the kernel

$$
\mathcal{E}_{v_{0}}\left(t ;\left(v_{1}, y_{1}\right),\left(v_{2}, y_{2}\right)\right)=2 \epsilon \alpha^{\prime}\left(\epsilon v_{0}\right) e_{\partial_{v}}\left(t ; v_{1}, v_{2}\right)\left(e_{v_{0}} * T e_{v_{0}}\right)\left(t ; y_{1}, y_{2}\right)
$$


2民-T. BENAMEUR, A. L. CAREY, J. PHILliPS, A. RENNIE, F. A. SUKOCHEV, AND K. P. WOJCIECHOWSKI

where $e_{\partial_{v}}\left(t ; v_{1}, v_{2}\right)$ denotes the kernel of the 1-dimensional heat operator defined on $\mathbf{R}$ by the operator $-\partial_{v}^{2}$. Let us also point out that $\epsilon \alpha^{\prime}\left(\epsilon v_{0}\right) T$ is simply equal to $\epsilon \dot{B}$ (at $v_{0}$ ), where $d o t$ denotes the derivative with respect to $u$-variable, so it is the operator $\left.\frac{d}{d v} B_{\epsilon v}\right|_{v=v_{0}}$.

At last, we are ready to formulate the $E A F$

Theorem 8.2. For any $t>0$ there exists $\epsilon_{0}$ and a constant $c>0$ such that for any $0<\epsilon<\epsilon_{0}$

$$
\frac{1}{\epsilon} \cdot\left\|k_{\epsilon}\left(t ;\left(v_{0}, y_{1}\right),\left(v_{0}, y_{2}\right)\right)-\mathcal{E}_{v_{0}}\left(t ;\left(v_{0}, y_{1}\right),\left(v_{0}, y_{2}\right)\right)\right\| \leq c \cdot \sqrt{\frac{\epsilon}{t}} .
$$

Remarks 8.3. (1) It has been already pointed out that we only present the proof of EAF for the family (8.3). The method we use works for any family $\left\{B_{u}\right\}_{0 \leq u \leq 1}$, such that for any $0 \leq u \leq 1$ the difference $B_{u}-B_{0}$ is an operator of order 0 and

$$
B_{1}=g B_{0} g^{-1} \text { and }\left\|B_{u}-B_{0}\right\| \leq c \cdot u \text {. }
$$

If the difference betweeen the operators $B_{u}$ is a 1 st order operator then we have to follow a more complicated version of the argument as presented in Cheeger's work [22].

(2) The proof we use allows us to replace $\sqrt{\epsilon}$, which appears on the right side of (8.8), by $\epsilon^{r}$ , for any $0<r<1$.

Proof. (2nd Part). The most technical part of the proof is presented in the next subsection. There we use the Duhamel's Principle to obtain the equality

$$
\begin{gathered}
e^{-t \Delta_{1, \epsilon}}-e^{-t \Delta_{2, \epsilon}}=\int_{0}^{t} e^{-s \Delta_{1, \epsilon}}\left(\Delta_{2, \epsilon}-\Delta_{1, \epsilon}\right) e^{-(t-s) \Delta_{2, \epsilon}} d s \\
=\int_{0}^{t}\left[e^{-s \Delta_{0}}\left(\Delta_{2, \epsilon}-\Delta_{1, \epsilon}\right) e^{-(t-s) \Delta_{0}}+\left(e^{-s \Delta_{1, \epsilon}}-e^{-s \Delta_{0}}\right)\left(\Delta_{2, \epsilon}-\Delta_{1, \epsilon}\right) e^{-(t-s) \Delta_{2, \epsilon}}\right] d s \\
+\int_{0}^{t} e^{-s \Delta_{0}}\left(\Delta_{2, \epsilon}-\Delta_{1, \epsilon}\right)\left(e^{-(t-s) \Delta_{2, \epsilon}}-e^{-(t-s) \Delta_{0}}\right) d s
\end{gathered}
$$

and we show that the kernels of the second and third terms on the right side are point-wise at most of the size $\epsilon^{\frac{3}{2}}$. Hence, we only have to study the first term and show that it gives the kernel (8.7) as $\epsilon \rightarrow 0$.

The operator

$$
\int_{0}^{t} e^{-s \Delta_{0}}\left(\Delta_{2, \epsilon}-\Delta_{1, \epsilon}\right) e^{-(t-s) \Delta_{0}} d s
$$

can be represented as

$$
\begin{aligned}
& \int_{0}^{t} e^{-s\left(-\partial_{v}^{2}\right)} e^{-s B_{\epsilon v_{0}}^{2}}\left(2 \epsilon \alpha^{\prime}(\epsilon v) T\right) e^{-(t-s)\left(-\partial_{v}^{2}\right)} e^{-(t-s) B_{\epsilon v_{0}}^{2}} d s=
\end{aligned}
$$

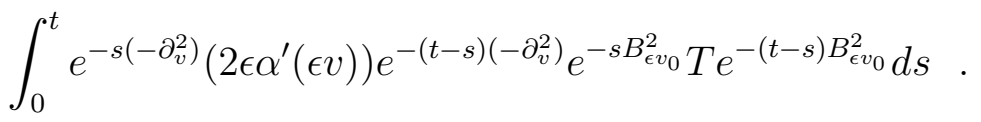


First, we study $l_{\epsilon}\left(s, t ; v_{1}, v_{2}\right)$ the kernel of the part which acts in the normal direction

$$
e^{-s\left(-\partial_{v}^{2}\right)}\left(2 \epsilon \alpha^{\prime}(\epsilon v)\right) e^{-(t-s)\left(-\partial_{v}^{2}\right)}
$$

We have

$$
l_{\epsilon}\left(s, t ; v_{0}, v_{0}\right)=\int_{-\infty}^{+\infty} \frac{e^{-\frac{\left(v_{0}-z\right)^{2}}{4 s}}}{\sqrt{4 \pi s}}\left(2 \epsilon \alpha^{\prime}(\epsilon z)\right) \frac{e^{-\frac{\left(v_{0}-z\right)^{2}}{4(t-s)}}}{\sqrt{4 \pi(t-s)}} d z .
$$

To simplify, we assume $v_{0}=0$ (this will be justified in the next subsection). We obtain

$$
l_{\epsilon}\left(s, t ; v_{0}, v_{0}\right)=\frac{1}{2 \pi} \int_{-\infty}^{+\infty} \frac{e^{-\frac{t z^{2}}{4 s(t-s)}}}{\sqrt{s(t-s)}}\left(\epsilon \alpha^{\prime}(\epsilon z)\right) d z .
$$

Now, we only have to show that

$$
\frac{1}{2 \pi} \int_{-\infty}^{+\infty} \frac{e^{-\frac{t z^{2}}{4 s(t-s)}}}{\sqrt{s(t-s)}}\left(\epsilon \alpha^{\prime}(\epsilon z)-\epsilon \alpha^{\prime}(0)\right) d z \mid \leq c \frac{\epsilon^{\frac{3}{2}}}{\sqrt{t}}
$$

This is the place where we use (8.2). We apply here the special case of a trick used in the next subsection.

First, an elementary computation shows that

$$
\int_{|z|>\frac{1}{\sqrt{\epsilon}}} \frac{e^{-\frac{t z^{2}}{4 s(t-s)}}}{\sqrt{s(t-s)}}\left(\epsilon \alpha^{\prime}(\epsilon z)-\epsilon \alpha^{\prime}(0)\right) d z \mid
$$

is exponentially small with respect to $\epsilon$. We have

$$
\begin{gathered}
\left|\int_{|z|>\frac{1}{\sqrt{\epsilon}}} \frac{e^{-\frac{t z^{2}}{4 s(t-s)}}}{\sqrt{s(t-s)}}\left(\epsilon \alpha^{\prime}(\epsilon z)-\epsilon \alpha^{\prime}(0)\right) d z\right| \leq c \frac{\epsilon^{\frac{3}{2}}}{\sqrt{t}} \mid \leq \epsilon c_{1} \cdot \int_{|z|>\frac{1}{\sqrt{\epsilon}}} \frac{e^{-\frac{t z^{2}}{4 s(t-s)}}}{\sqrt{s(t-s)}} d z \\
=\frac{2}{\sqrt{t}} \cdot \int_{r>\sqrt{\frac{t}{4 s(t-s) \epsilon}}} e^{-r^{2}} \leq \frac{2}{\sqrt{t}} \cdot e^{-\frac{1}{4 t \epsilon}} \leq c_{1} e^{-\frac{c_{2}}{t \epsilon}} .
\end{gathered}
$$

It follows now from (8.2) that

$$
\left|\alpha^{\prime}(\epsilon z)-\alpha^{\prime}(0)\right| \leq c_{3} \sqrt{\epsilon}
$$

for $z \leq \frac{1}{\sqrt{\epsilon}}$. This gives

$$
\left|\int_{|z|<\frac{1}{\sqrt{\epsilon}}} \frac{e^{-\frac{t z^{2}}{4 s(t-s)}}}{\sqrt{s(t-s)}}\left(\epsilon \alpha^{\prime}(\epsilon z)-\epsilon \alpha^{\prime}(0)\right) d z\right| \leq c_{3} \epsilon^{\frac{3}{2}} \cdot \int_{-\infty}^{+\infty} \frac{e^{-\frac{t z^{2}}{4 s(t-s)}}}{\sqrt{s(t-s)}} \leq c_{4} \frac{\epsilon^{\frac{3}{2}}}{\sqrt{t}} .
$$

We see that up to a term of order $\frac{\epsilon^{\frac{3}{2}}}{\sqrt{t}}$ the kernel $l_{\epsilon}\left(s, t ; v_{1}, v_{2}\right)\left(\right.$ for $\left.v_{1}=v_{2}=v_{0}\right)$ is equal to

$$
\epsilon \alpha^{\prime}\left(v_{0}\right) \frac{1}{2 \pi} \int_{-\infty}^{+\infty} \frac{e^{-\frac{t z^{2}}{4 s(t-s)}}}{\sqrt{s(t-s)}}=\frac{\epsilon \alpha^{\prime}\left(v_{0}\right)}{\sqrt{t}}
$$


2M-T. BENAMEUR, A. L. CAREY, J. PHILLIPS, A. RENNIE, F. A. SUKOCHEV, AND K. P. WOJCIECHOWSKI and $l_{\epsilon}\left(s, t ; v_{1}, v_{2}\right)$ can be replaced by the kernel of the operator

$$
2 \epsilon \alpha^{\prime}\left(v_{0}\right) e^{-t\left(-\partial_{v}^{2}\right)} \text {. }
$$

As a result of these estimates the operator (8.9) can be replaced by

$$
2 \epsilon \alpha^{\prime}\left(\epsilon v_{0}\right) e^{-t\left(-\partial_{v}^{2}\right)} \int_{0}^{t} e^{-s B_{\epsilon v_{0}}^{2}} T e^{-(t-s) B_{\epsilon v_{0}}^{2}} d s
$$

which has kernel equal to

$$
2 \epsilon \alpha^{\prime}\left(\epsilon v_{0}\right) e_{\partial_{v}}\left(t ; v_{1}, v_{2}\right) \int_{0}^{t} d s \int_{M} e_{v_{0}}\left(s ; y_{1}, w\right) T(w) e_{v_{0}}\left(t-s ; w, y_{2}\right) d w
$$

which is exactly the kernel $\mathcal{E}_{v_{0}}$.

Corollary 8.4. The spectral flow formula (8.4) follows from the EAF.

Proof. The EAF shows that $k_{\epsilon}\left(t ;\left(v_{1}, y_{1}\right),\left(v_{2}, y_{2}\right)\right)$ is equal to

$$
2 \epsilon \alpha^{\prime}\left(\epsilon v_{1}\right) e_{\partial_{v}}\left(t ; v_{1}, v_{2}\right)\left(e_{v_{1}} * T e_{v_{1}}\right)\left(t ; y_{1}, y_{2}\right)+O\left(\frac{\epsilon^{\frac{3}{2}}}{\sqrt{t}}\right)
$$

It follows that, for $\epsilon$ small enough, we have the equality

$$
\begin{gathered}
\text { index } \mathcal{D}=2 \int_{N_{\epsilon}} \operatorname{tr} \epsilon \alpha^{\prime}\left(\epsilon v_{0}\right) e_{\partial_{v}}\left(t ; v_{0}, v_{0}\right)\left(e_{v_{0}} * T e_{v_{0}}\right)(t ; y, y) d y d v_{0} \\
=\int_{0}^{\frac{1}{\epsilon}} \frac{\epsilon \alpha^{\prime}\left(\epsilon v_{0}\right)}{\sqrt{\pi t}} d v_{0} \int_{0}^{t} \operatorname{Tr}_{M} e^{-s B_{\epsilon v_{0}}^{2} T e^{-(t-s) B_{\epsilon v_{0}}^{2}} d s} \\
=\int_{0}^{\frac{1}{\epsilon}} \frac{\epsilon \alpha^{\prime}\left(\epsilon v_{0}\right)}{\sqrt{\pi t}} d v_{0} \int_{0}^{t} \operatorname{Tr}_{M} T e^{-t B_{\epsilon v_{0}}^{2}} d s \\
=\sqrt{\frac{t}{\pi}} \int_{0}^{\frac{1}{\epsilon}} \alpha^{\prime}\left(\epsilon v_{0}\right) \operatorname{Tr}_{M} T e^{-t B_{\epsilon v_{0}}^{2}}\left(\epsilon d v_{0}\right)=\sqrt{\frac{t}{\pi}} \int_{0}^{1} \operatorname{Tr}_{M} \dot{B}_{u} e^{-t B_{u}^{2}} d u
\end{gathered}
$$

Let us observe that (for compatible Dirac operators)

$$
\lim _{t \rightarrow 0} \sqrt{\frac{t}{\pi}} \cdot \int_{0}^{1} \operatorname{Tr}_{M} \dot{B}_{u} e^{-t B_{u}^{2}} d u=\int_{0}^{1} d u \lim _{t \rightarrow 0} \sqrt{\frac{t}{\pi}} \cdot \operatorname{Tr}_{M} \dot{B}_{u} e^{-t B_{u}^{2}}=\int_{0}^{1} \dot{\eta}_{u} d u
$$

where $\eta_{u}=\frac{1}{2}\left(\operatorname{dim} \operatorname{ker}\left(B_{u}\right)+\eta_{B_{u}}(0)\right)$ is the $\eta$-invariant of the operator $B_{u}[3]$. 
8.3. Technicalities. We write the operator $\mathcal{D}_{\epsilon}$ as

$$
\mathcal{D}_{\epsilon}=\partial_{v}+\left(B_{0}+\alpha\left(\epsilon v_{0}\right) T\right)+\left(\alpha(\epsilon v)-\alpha\left(\epsilon v_{0}\right)\right) T=\partial_{v}+B_{\epsilon v_{0}}+\beta(\epsilon v) T .
$$

In the following we consider the operator $\mathcal{D}_{\epsilon}$ as an operator living on $\mathbf{R} \times M$. This does not change anything in the proof, but simplifies the computations. Of course there is a problem with the definition of the index of $\mathcal{D}_{\epsilon}$ in this set-up. Even though $B_{\epsilon v}$ is a constant operator for $v<0$ and $\frac{1}{\epsilon} \leq v$ the index may not be well-defined unless the operator $B_{\epsilon v}$ is invertible for those values of the normal coordinate. Hence, one can think that we perturbed the tangential operator by a small number and the invertibility condition is satisfied. In any case it follows that the integral from the kernel $k_{\epsilon}(t ;(v, y),(v, y))$ over $\left[0, \frac{1}{\epsilon}\right] \times M$ gives an integer equal to the index of $\mathcal{D}_{\epsilon}$ on $N_{\epsilon}$ and the integral over the leftover of $\mathbf{R} \times M$ gives a finite error term, which goes to 0 as $\epsilon \rightarrow 0$. Hence it is not difficult to show that

$$
\text { index } \mathcal{D}_{\epsilon}=\int_{\mathbf{R} \times M} \operatorname{tr} k_{\epsilon}(t ;(v, y),(v, y)) d y d v,
$$

where on the left side we have the operator on $N_{\epsilon}$.

We work on $\mathbf{R} \times M$ and we have to show that $E A F$ holds at any given point $\left(v_{0}, y\right)$. After reparametrization, we can assume that $v_{0}=0$ and our operator has the form

$$
\mathcal{D}_{\epsilon}=\partial_{v}+B_{0}+\beta(\epsilon v) T
$$

where the cut-off function $\beta(v)$ satisfies $\beta(0)=0$. The corresponding Laplacians are

$$
\begin{gathered}
\Delta_{1, \epsilon}=\mathcal{D}_{\epsilon}^{*} \mathcal{D}_{\epsilon}=-\partial_{v}^{2}+B_{0}^{2}+\beta(\epsilon v)\left(B_{0} T+T B_{0}\right)+\beta^{2}(\epsilon v) T^{2}-\epsilon \beta^{\prime}(\epsilon v) T \\
=-\partial_{v}^{2}+B_{0}^{2}+\beta(\epsilon v) T_{1}-\epsilon \beta^{\prime}(\epsilon v) T
\end{gathered}
$$

and

$$
\Delta_{2, \epsilon}=\mathcal{D}_{\epsilon} \mathcal{D}_{\epsilon}^{*}=-\partial_{v}^{2}+B_{0}^{2}+\beta(\epsilon v) T_{1}+\epsilon \beta^{\prime}(\epsilon v) T
$$

where $T_{1}$ denotes the 1 st order tangential operator $B_{0} T+T B_{0}+\beta(\epsilon v) T^{2}$.

To evaluate $k_{\epsilon}(t ;(0, y),(0, y))$ we apply Duhamel's Principle [51, 10]:

$$
\begin{gathered}
e^{-t \Delta_{1, \epsilon}}-e^{-t \Delta_{2, \epsilon}}=\int_{0}^{t} \frac{d}{d s}\left(e^{-s \Delta_{1, \epsilon}} e^{-(t-s) \Delta_{2, \epsilon}}\right) d s \\
=\int_{0}^{t} e^{-s \Delta_{1, \epsilon}}\left(\Delta_{2, \epsilon}-\Delta_{1, \epsilon}\right) e^{-(t-s) \Delta_{2, \epsilon}} d s .
\end{gathered}
$$

The difference $\Delta_{2, \epsilon}-\Delta_{1, \epsilon}$ is equal to the bundle endomorphism $2 \epsilon \beta^{\prime}(\epsilon v) T$ and this is the term which brings the 1st, and the most important, factor of $\epsilon$ into the formula. Once again, we simplify the presentation and introduce Laplacian $\Delta_{0}=-\partial_{v}^{2}+B_{0}^{2}$ and study each summand in the equality

$$
e^{-t \Delta_{1, \epsilon}}-e^{-t \Delta_{2, \epsilon}}=\left(e^{-t \Delta_{1, \epsilon}}-e^{-t \Delta_{0}}\right)+\left(e^{-t \Delta_{0}}-e^{-t \Delta_{2, \epsilon}}\right)
$$

The application of Duhamel's Principle to the first summand leads to the series

$$
e^{-t \Delta_{1, \epsilon}}-e^{-t \Delta_{0}}=\int_{0}^{t} e^{-s \Delta_{1, \epsilon}}\left(\Delta_{0}-\Delta_{1, \epsilon}\right) e^{-(t-s) \Delta_{0}} d s
$$


2\$-T. BENAMEUR, A. L. CAREY, J. PHILliPS, A. RENNIE, F. A. SUKOCHEV, AND K. P. WOJCIECHOWSKI

$$
\begin{gathered}
\int_{0}^{t} e^{-s_{1} \Delta_{0}}\left(\Delta_{0}-\Delta_{1, \epsilon}\right) e^{-\left(t-s_{1}\right) \Delta_{0}} d s_{1} \\
+\int_{0}^{t} d s_{1} \int_{0}^{s_{1}} d s_{2} e^{-s_{2} \Delta_{1, \epsilon}}\left(\Delta_{0}-\Delta_{1, \epsilon}\right) e^{-\left(s_{1}-s_{2}\right) \Delta_{0}}\left(\Delta_{0}-\Delta_{1, \epsilon}\right) e^{-\left(t-s_{1}\right) \Delta_{0}} \\
=\sum_{k=1}^{\infty} \int_{0}^{t} d s_{1} \ldots \int_{0}^{s_{k-1}} d s_{k} e^{-s_{k} \Delta_{0}}\left(\Delta_{0}-\Delta_{1, \epsilon}\right) \ldots\left(\Delta_{0}-\Delta_{1, \epsilon}\right) e^{-\left(t-s_{1}\right) \Delta_{0}} .
\end{gathered}
$$

The result we state now is a standard application of Duhamel's Principle

Proposition 8.5. Let us consider the operator $\Delta_{R}=\Delta_{0}+\beta(\epsilon v) R$, where $R: C^{\infty}(M ; S) \rightarrow$ $C^{\infty}(M ; S)$ is a tangential differential operator of order 1 . Then, there exists positive constants $c_{1}$ and $c_{2}$ (independent of $\epsilon$ ) such that for any sufficiently small $\epsilon$ the following estimate holds

$$
\left\|e_{R}\left(t ;\left(v_{1}, y_{1}\right),\left(v_{2}, y_{2}\right)\right)\right\| \leq c_{1} t^{-\frac{m+1}{2}} e^{-c_{2}} \frac{d^{2}\left(\left(\left(v_{1}, y_{1}\right),\left(v_{2}, y_{2}\right)\right)\right.}{2},
$$

where $e_{R}\left(t ;\left(v_{1}, y_{1}\right),\left(v_{2}, y_{2}\right)\right)$ denotes the kernel of $e^{-t \Delta_{R}}$.

Proof. The estimate (8.11) holds for $\Delta_{0}$ and we have

$$
\begin{gathered}
e^{-t \Delta_{R}}-e^{-t \Delta_{0}}=\sum_{k=1}^{\infty} \int_{0}^{t} d s_{1} \ldots \int_{0}^{s_{k-1}} d s_{k} e^{-s_{k} \Delta_{0}}(\beta(\epsilon v) R) \ldots(\beta(\epsilon v) R) e^{-\left(t-s_{1}\right) \Delta_{0}} \\
=\sum_{k=1}^{\infty} \int_{0}^{t} d s_{1} \ldots \int_{0}^{s_{k-1}} d s_{k}\left(e^{-s_{k}\left(-\partial_{v}^{2}\right)}(\beta(\epsilon v)) \ldots . .(\beta(\epsilon v)) e^{-\left(t-s_{1}\right)\left(-\partial_{v}^{2}\right)}\right) \times \\
\left(e^{-s_{k} B_{0}^{2}} R \ldots . R e^{-\left(t-s_{1}\right) B_{0}^{2}}\right) .
\end{gathered}
$$

The kernel of the operator in the first bracket is estimated as follows

$$
\begin{gathered}
\left\|\left(e^{-s_{k}\left(-\partial_{v}^{2}\right)}(\beta(\epsilon v)) \ldots . .(\beta(\epsilon v)) e^{-\left(t-s_{1}\right)\left(-\partial_{v}^{2}\right)}\right)\left(t ; v_{1}, v_{2}\right)\right\| \leq \\
\left\|\left(e^{-s_{k}\left(-\partial_{v}^{2}\right)} \ldots e^{-\left(t-s_{1}\right)\left(-\partial_{v}^{2}\right)}\right)\left(t ; v_{1}, v_{2}\right)\right\|,
\end{gathered}
$$

as $0 \leq \beta(\epsilon v)$. This leads to the estimate

$$
\begin{gathered}
\left\|e_{R}\left(t ;\left(v_{1}, y_{1}\right),\left(v_{2}, y_{2}\right)\right)-e_{0}\left(t ;\left(v_{1}, y_{1}\right),\left(v_{2}, y_{2}\right)\right)\right\| \leq \\
\frac{e^{-\frac{\left(v_{1}-v_{2}\right)^{2}}{4 t}}}{\sqrt{4 \pi t}} \sum_{k=1}^{\infty}\left\|\left(e^{-s_{k} B_{0}^{2}} R \ldots . R e^{-\left(t-s_{1}\right) B_{0}^{2}}\right)\left(t ; y_{1}, y_{2}\right)\right\| .
\end{gathered}
$$

The series here is estimated in the standard way. The kernel of the operator $R e^{-t B_{0}^{2}}$ is bounded by $c_{1} t^{-\frac{m+1}{2}} e^{-c_{2}} \frac{\left(v_{1}-v_{2}\right)^{2}}{t}$. Therefore we follow [51] and obtain

$$
\sum_{k=1}^{\infty}\left\|\left(e^{-s_{k} B_{0}^{2}} R \ldots R e^{-\left(t-s_{1}\right) B_{0}^{2}}\right)\left(t ; y_{1}, y_{2}\right)\right\| \leq c_{3} t^{-\frac{m-1}{2}} e^{-c_{4} \frac{d^{2}\left(y_{1}, y_{2}\right)}{t}} .
$$


The positive constants above do not depend on $\epsilon$.

It is no problem to see that the estimate (8.11) from Proposition 8.5] is also satisfied by kernels of the heat operators defined by $\Delta_{1, \epsilon}$ and $\Delta_{2, \epsilon}$, which leads to the following useful property.

Corollary 8.6. The contribution to the kernel $k_{\epsilon}\left(t ;\left(0, y_{1}\right),\left(0, y_{2}\right)\right)$ provided by the points distant more than $\frac{1}{\sqrt{\epsilon}}$ from $\{0\} \times M$ may be disregarded.

Proof. We have

$$
\begin{gathered}
\left\|\left(e^{-t \Delta_{1, \epsilon}}-e^{-t \Delta_{2, \epsilon}}\right)\left(t ;\left(0, y_{1}\right),\left(0, y_{2}\right)\right)\right\|= \\
\left\|\int_{0}^{t} e^{-s \Delta_{1, \epsilon}}(2 \epsilon \beta(\epsilon v) T) e^{-(t-s) \Delta_{2, \epsilon}}\left(t ;\left(0, y_{1}\right),\left(0, y_{2}\right)\right) d s\right\| \leq \\
\int_{0}^{t} d s \int_{\mathbf{R} \times M} d u d z\left\|e_{1, \epsilon}\left(s ;\left(0, y_{1}\right),(u, z)\right) 2 \epsilon \beta(\epsilon u) T(u) e_{2, \epsilon}(t-s ;(u, z),(0, y))\right\| .
\end{gathered}
$$

We want to show that the integral over $|u|>\frac{1}{\sqrt{\epsilon}}$ is exponentially small with respect to $\epsilon$.

$$
\begin{gathered}
\int_{0}^{t} d s \int_{|u|>\frac{1}{\sqrt{\epsilon}}} d u \int_{M} d z\left\|e_{1, \epsilon}\left(s ;\left(0, y_{1}\right),(u, z)\right)\right\| 2 \epsilon \beta(\epsilon u) \times \\
\times\|T(u)\|\left\|e_{2, \epsilon}(t-s ;(u, z),(0, y))\right\| \\
\leq c_{3} \epsilon \int_{0}^{t} d s \int_{|u|>\frac{1}{\sqrt{\epsilon}}} d u \int_{M} d z c_{1} s^{-\frac{m+1}{2}} e^{-c_{2} \frac{d^{2}\left(\left(0, y_{1}\right),(u, z)\right)}{s}}(t-s)^{-\frac{m+1}{2}} \times \\
\leq c_{4} \epsilon \cdot \operatorname{vol}(M) \int_{0}^{t} d s \int_{|u|>\frac{1}{\sqrt{\epsilon}}}^{-c_{2}} \frac{{\frac{d}{2}\left((u, z),\left(0, y_{2}\right)\right)}_{t-s}}{(s(t-s))^{-\frac{m+1}{2}} e^{-c_{2} \frac{u^{2}}{s}} e^{-c_{2}} \frac{u^{2}}{t-s} d u} \\
\leq c_{4} \epsilon \cdot \operatorname{vol}(M) \int_{0}^{t} d s \int_{|u|>\frac{1}{\sqrt{\epsilon}}} e^{-c_{5} \frac{u^{2}}{s}} e^{-c_{5} \frac{u^{2}}{t-s}} d u \\
\leq c_{4} \epsilon \cdot \operatorname{vol}(M) \int_{0}^{t} d s \int_{|u|>\frac{1}{\sqrt{\epsilon}}} e^{-c_{5} \frac{u^{2}}{t}} d u \leq c_{6} \epsilon \cdot e^{-\frac{c_{7}}{\epsilon t}} .
\end{gathered}
$$

Now the idea of the proof of the $E A F$ can be easily understood. The kernel $k_{\epsilon}\left(t ;\left(0, y_{1}\right),\left(0, y_{2}\right)\right.$ is the kernel of the operator

$$
\int_{0}^{t} e^{-s \Delta_{1, \epsilon}}\left(\Delta_{2, \epsilon}-\Delta_{1, \epsilon}\right) e^{-(t-s) \Delta_{2, \epsilon}} d s
$$


Kernels of both heat operators $e^{-t \Delta_{i, \epsilon}}$, expands into series where the leading term is the kernel of the operator $e^{-t \Delta_{0}}$. It follows that the leading term in the expansion of the kernel of the operator (8.12) is

$$
\int_{0}^{t} d s \int_{\mathbf{R} \times M} e_{0}\left(s ;\left(0, y_{1}\right),(z, w)\right)\left(2 \epsilon \beta^{\prime}(\epsilon z) T(w)\right) e_{0}\left(t-s ;(z, w),\left(0, y_{2}\right)\right) d z d w .
$$

This gives us the contribution which appears in the $E A F$.

We have to show that further perturbation brings the consecutive powers of $\sqrt{\epsilon}$ into the picture in order to finish the proof. So let us replace $e_{0}\left(s ;\left(0, y_{1}\right),(z, w)\right)$ by $e_{1, \epsilon}\left(s ;\left(0, y_{1}\right),(z, w)\right)$ in the formula (8.13), hence

$$
\int_{0}^{t} e^{-s \Delta_{0}}\left(2 \epsilon \beta^{\prime}(\epsilon z) T(w)\right) e^{-(t-s) \Delta_{0}} d s
$$

is replaced by

$$
\int_{0}^{t} e^{-s \Delta_{1, \epsilon}}\left(2 \epsilon \beta^{\prime}(\epsilon z) T(w)\right) e^{-(t-s) \Delta_{0}} d s
$$

and the kernel of $e^{-s \Delta_{1, \epsilon}}-e^{-s \Delta_{0}}$ brings the extra factor $\sqrt{\epsilon}$ as the elementary estimates presented below show. The operator $\Delta_{1, \epsilon}$ is obtained from $\Delta_{0}$ by adding the correction term of the form $\gamma(\epsilon v) S_{1}$, where $\gamma(\epsilon v)$ is the cut-off function with the properties specified earlier.

\section{The main estimate}

We have

$$
\begin{gathered}
\left\|e_{1, \epsilon}(t ;(0, y),(0, w))-e_{0}(t ;(0, y),(0, q))\right\| \\
=\left\|\int_{0}^{t} d s \int_{|v| \leq \sqrt{\epsilon}} d v \int_{M} d z e_{1, \epsilon}(s ;(0, y),(v, z)) \gamma(\epsilon v) S_{1}(z) e_{0}(t ;(v, z),(0, q))\right\| \\
\leq \int_{0}^{t} d s \int_{-\frac{1}{\sqrt{\epsilon}}}^{-\frac{1}{\sqrt{\epsilon}}} d v \int_{M} d z\left\|e_{1, \epsilon}(s ;(0, y),(v, z))\right\| \cdot|\gamma(\epsilon v)| \cdot\left\|S_{1}(z) e_{0}(t ;(v, z),(0, q))\right\| \\
\leq c \sqrt{\epsilon} \int_{0}^{t} d s \int_{-\frac{1}{\sqrt{\epsilon}}}^{-\frac{1}{\sqrt{\epsilon}}} d v \int_{M} d z c_{1} s^{-\frac{m+1}{2}} e^{-c_{2} \frac{v^{2}+d^{2}(y, z)}{s}} c_{1}(t-s)^{-\frac{m+2}{2}} e^{-c_{2} \frac{v^{2}+d^{2}(z, q)}{t-s}} \\
\leq c \sqrt{\epsilon} c_{1}^{2} \int_{0}^{t} \frac{1}{\sqrt{t-s}} d s \int_{-\frac{1}{\sqrt{\epsilon}}}^{-\frac{1}{\sqrt{\epsilon}}} d v \frac{e^{-c_{2} \frac{t v^{2}}{s(t-s)}}}{\sqrt{s(t-s)}} \int_{M} d z \frac{e^{-c_{2} \frac{d^{2}(y, z)}{s}} e^{-c_{2} \frac{d^{2}(z, q)}{t-s}}}{(s(t-s))^{-\frac{m}{2}}} \\
\leq c_{3} \frac{\sqrt{\epsilon}}{\sqrt{t}} \cdot \int_{0}^{t} \frac{1}{\sqrt{t-s}} d s \int_{M} d z(s(t-s))^{-\frac{m}{2}} e^{-c_{2} \frac{d^{2}(y, z)}{s}} e^{-c_{2} \frac{d^{2}(z, q)}{t-s}} .
\end{gathered}
$$

The following elementary inequality is used to estimate the factor $e^{-c_{2} \frac{d^{2}(y, z)}{s}} e^{-c_{2} \frac{d^{2}(z, q)}{t-s}}$,

$$
\frac{d^{2}(y, q)}{t} \leq \frac{d^{2}(y, z)}{s}+\frac{d^{2}(z, q)}{t-s} .
$$


We have

$$
\begin{gathered}
e^{-c_{2} \frac{d^{2}(y, z)}{s}} e^{-c_{2} \frac{d^{2}(z, q)}{t-s}}=e^{-2 c_{2} \frac{d^{2}(y, z)}{2 s}} e^{-2 c_{2} \frac{d^{2}(z, q)}{2(t-s)}} \leq \\
e^{-c_{2} \frac{d^{2}(y, q)}{2 t}} e^{-c_{2} \frac{d^{2}(y, z)}{2 s}} e^{-c_{2} \frac{d^{2}(z, q)}{2(t-s)}} .
\end{gathered}
$$

All this amounts to

$$
\begin{gathered}
\left\|e_{R}(t ;(0, y),(0, w))-e_{0}(t ;(0, y),(0, q))\right\| \\
\leq c_{3} \sqrt{\frac{\epsilon}{t}} \cdot e^{-c_{2} \frac{d^{2}(y, q)}{2 t}} \int_{0}^{t} \frac{d s}{\sqrt{t-s}} \int_{M} d z(s(t-s))^{-\frac{m}{2}} e^{-c_{2} \frac{d^{2}(y, z)}{2 s}} e^{-c_{2} \frac{d^{2}(z, q)}{2(t-s)}} \\
\leq c_{4} \sqrt{e} \cdot e^{-c_{2} \frac{d^{2}(y, q)}{2 t}} \int_{M} d z(s(t-s))^{-\frac{m}{2}} e^{-c_{2} \frac{d^{2}(y, z)}{2 s}} e^{-c_{2} \frac{d^{2}(z, q)}{2(t-s)}} \\
\leq c_{5} \sqrt{e} \cdot t^{-\frac{m}{2}} \cdot e^{-c_{2} \frac{d^{2}(y, q)}{2 t}} .
\end{gathered}
$$

Now, we can show the fact used in the proof of the $E A F$ in the previous subsection. We have

$$
\begin{gathered}
e^{-t \Delta_{1, \epsilon}}-e^{-t \Delta_{2, \epsilon}}=\int_{0}^{t} e^{-s \Delta_{1, \epsilon}}\left(\Delta_{2, \epsilon}-\Delta_{1, \epsilon}\right) e^{-(t-s) \Delta_{2, \epsilon}} d s \\
=\int_{0}^{t}\left[e^{-s \Delta_{0}}\left(\Delta_{2, \epsilon}-\Delta_{1, \epsilon}\right) e^{-(t-s) \Delta_{2, \epsilon}}+\left(e^{-s \Delta_{1, \epsilon}}-e^{-s \Delta_{0}}\right)\left(\Delta_{2, \epsilon}-\Delta_{1, \epsilon}\right) e^{-(t-s) \Delta_{2, \epsilon}}\right] d s \\
=\int_{0}^{t} e^{-s \Delta_{0}}\left(\Delta_{2, \epsilon}-\Delta_{1, \epsilon}\right) e^{-(t-s) \Delta_{0}} d s \\
+\int_{0}^{t}\left(e^{-s \Delta_{1, \epsilon}}-e^{-s \Delta_{0}}\right)\left(\Delta_{2, \epsilon}-\Delta_{1, \epsilon}\right) e^{-(t-s) \Delta_{2, \epsilon}} d s \\
+\int_{0}^{t} e^{-s \Delta_{0}}\left(\Delta_{2, \epsilon}-\Delta_{1, \epsilon}\right)\left(e^{-(t-s) \Delta_{2, \epsilon}}-e^{-(t-s) \Delta_{0}}\right) d s .
\end{gathered}
$$

The operator $\Delta_{2, \epsilon}-\Delta_{1, \epsilon}=2 \epsilon \beta^{\prime}(\epsilon v) T$ brings a factor $\epsilon$ and the first term on the right side is of the form $\int_{0}^{t} e^{-s \Delta_{0}}\left(\Delta_{2, \epsilon}-\Delta_{1, \epsilon}\right) e^{-(t-s) \Delta_{0}} d s$ and of the $\epsilon$ size. The next term on the right side

$$
\int_{0}^{t}\left(e^{-s \Delta_{1, \epsilon}}-e^{-s \Delta_{0}}\right)\left(\Delta_{2, \epsilon}-\Delta_{1, \epsilon}\right) e^{-(t-s) \Delta_{2, \epsilon}} d s
$$

contains additionally the difference $e^{-s \Delta_{1, \epsilon}}-e^{-s \Delta_{0}}$, hence it is of the size $\epsilon^{\frac{3}{2}}$. This is also the case of the last term $\int_{0}^{t} e^{-s \Delta_{0}}\left(\Delta_{2, \epsilon}-\Delta_{1, \epsilon}\right)\left(e^{-(t-s) \Delta_{2, \epsilon}}-e^{-(t-s) \Delta_{0}}\right) d s$. It follows that, as we take limit as $\epsilon \rightarrow 0$, only the integral (over $\mathbf{R} \times M$ ) from the first term is going to survive. The kernel of this operator at the point $\left(t,\left(0, y_{1}\right),\left(0, y_{2}\right)\right)$ has the form

$$
\left(e_{0} \#\left(2 \epsilon \beta^{\prime}(\epsilon v) T e_{0}\right)\left(t,\left(0, y_{1}\right),\left(0, y_{2}\right)\right),\right.
$$

where $e_{0}\left(t ;\left(v_{1}, y_{1}\right),\left(v_{2}, y_{2}\right)\right)$ denotes kernel of the operator $e^{-t B_{0}^{2}}$. This is exactly what we need in order to complete the proof. 
8.4. The EAF for operators on covering spaces. We let $\tilde{M}$ be the universal covering space for the closed manifold $M$, with the corresponding fundamental group $G\left(=\pi_{1}(M)\right)$. We assume that we are given a $G$-invariant, compatible Dirac operator on $\tilde{M}$

$$
\tilde{B}: C^{\infty}(\tilde{M} ; \tilde{S} \otimes \tilde{E}) \rightarrow C^{\infty}(\tilde{M} ; \tilde{S} \otimes \tilde{E})
$$

where $G$ acts on $\tilde{E}$ via a representation $\rho$. The appropriate von Neumann algebra $\mathcal{N}$ is the commutant of the $G$ action and there is a corresponding trace $\tau=\tau_{G}$ as described by Atiyah [1] and introduced at the beginning of this Section.

We introduce now a $G$-invariant unitary bundle automorphism $\tilde{h}$ of the auxiliary bundle $\tilde{E}$, and we consider family $\left\{\tilde{B}_{u}\right\}$ as defined in (8.1). This family has a well-defined spectral flow and we want to prove that

$$
\operatorname{sf}\left\{\tilde{B}_{s}\right\}=\operatorname{index} \tilde{A}
$$

(Here the tilde just denotes the covering space analogues of the operators we introduced before). This is the result which corresponds to Theorem 8.1

\section{Theorem 8.7.}

$$
\text { index } \tilde{A}=\operatorname{sf}\left\{\tilde{B}_{s}\right\}=\int_{0}^{1} \dot{\eta}_{s}^{G} d s .
$$

We remind the reader that the $\eta$-invariant in this context was studied by Cheeger and Gromov [23, Hurder [4] and later on by Mathai [4] and others. To prove the theorem 8.7 have only to show

$$
\operatorname{index}_{G} \tilde{A}=\int_{0}^{1} \sqrt{\frac{t}{\pi}} \cdot \tau_{G} \dot{B}_{s} e^{-t B_{s}^{2}} d s,
$$

This however follows easily from the extension of the EAF to the present context. We follow the previous argument. The $i n \operatorname{dex}_{G} \tilde{A}$ is equal to

$$
\tau_{G}\left(e^{-t \tilde{A}^{*} \tilde{A}}-e^{-t \tilde{A} \tilde{A}^{*}}\right)
$$

(The McKean-Singer formula for the index holds when the von Neumann algebra is not necessarily a factor [19]). We blow up the metric and all the arguments from the compact case come through with the slight modifications.

The only problem we face is that although our trace is defined by the integral over the (compact) fundamental domain we have to integrate over the whole non-compact manifold $\tilde{M}$, when applying Duhamel's Principle. First let us notice that the standard point-wise estimate on the heat-kernel (8.11) holds on $\tilde{M}$ [36. The difficulty follows from the well-known fact that the volume of the ball with a fixed center on $\tilde{M}$ may grow exponentially with the radius of the ball. Therefore we have to be careful with the arguments which lead to the proof of the results which correspond to Proposition 8.5 and Corollary 8.6. Actually, everything works only because the volume growth is at most exponential in the diameter. We omit the estimates which lead to the proof of Proposition 8.5 for the case of the covering space $\tilde{M}$. However to present the flavour of the computations involved we present a modification of the argument used to get 
Corollary 8.6. We work on the space $\tilde{M}_{\epsilon}$ (or on $\mathbf{R} \times \tilde{M}$ ) now, so the proper formulation of the result is as follows.

Corollary 8.8. Let $y_{1}, y_{2} \in \bar{M}$ then we may disregard the contribution to the kernel $k_{\epsilon}\left(t ;\left(0, y_{1}\right),\left(0, y_{2}\right)\right)$ provided by the points $(u, z) \in \mathbf{R} \times \tilde{M}$ such that $|u|>\frac{1}{\sqrt{\epsilon}}$ and $d(z, \bar{M})>1$.

Proof. We start as in Section 8.2

$$
\begin{gathered}
\left\|e^{-t \Delta_{1, \epsilon}}-e^{-t \Delta_{2, \epsilon}}\right\|\left(t ;\left(0, y_{1}\right),\left(0, y_{2}\right)\right)= \\
\left\|\int_{0}^{t} e^{-s \Delta_{1, \epsilon}}(2 \epsilon \beta(\epsilon v) T) e^{-(t-s) \Delta_{2, \epsilon}}\right\|\left(t ;\left(0, y_{1}\right),\left(0, y_{2}\right)\right) d s \leq \\
\int_{0}^{t} d s \int_{\mathbf{R} \times \tilde{M}} d u d z\left\|e_{1, \epsilon}\left(s ;\left(0, y_{1}\right),(u, z)\right) 2 \epsilon \beta(\epsilon u) T(u) e_{2, \epsilon}(t-s ;(u, z),(0, y))\right\| .
\end{gathered}
$$

We have to remember that nothing is changed (i.e. we obtain a negligible contribution), while we work on compact pieces of the space $\mathbf{R} \times \tilde{M}$. Here we want to show that the integral over $(u, z) \in \mathbf{R} \times \tilde{M}$ such that $|u|>\frac{1}{\sqrt{\epsilon}}$ and $d(z, \bar{M})>1$ is exponentially small with respect to $\epsilon$.

$$
\begin{gathered}
\int_{0}^{t} d s \int_{|u|>\frac{1}{\sqrt{\epsilon}}} d u \int_{\{z ; d(z, \bar{M})>1\}} d z \\
\left\|e_{1, \epsilon}\left(s ;\left(0, y_{1}\right),(u, z)\right)\right\| 2 \epsilon \beta(\epsilon u)\|T(u)\|\left\|e_{2, \epsilon}(t-s ;(u, z),(0, y))\right\| \leq \\
c_{3} \epsilon \cdot \int_{0}^{t} d s \int_{|u|>\frac{1}{\sqrt{\epsilon}}} d u \int_{\{z ; d(z, \bar{M})>1\}} d z e^{-c_{2} \frac{d^{2}\left(\left(0, y_{1}\right),(u, z)\right)}{s}} e^{-c_{2} \frac{d^{2}\left((u, z),\left(0, y_{2}\right)\right)}{t-s}} \leq \\
c_{3} \epsilon \cdot \int_{0}^{t} d s \int_{|u|>\frac{1}{\sqrt{\epsilon}}} e^{-c_{2} \frac{u^{2}}{s}} e^{-c_{2} \frac{u^{2}}{t-s}} d u \int_{\{z ; d(z, \bar{M})>1\}} d z e^{-c_{2} \frac{d^{2}(z ; \bar{M}}{s}} e^{-c_{2} \frac{d^{2}(z ; \bar{M}}{t-s}} .
\end{gathered}
$$

The first integral on the right side is estimated as in Section 2

$$
\begin{gathered}
\int_{|u|>\frac{1}{\sqrt{\epsilon}}} e^{-c_{2} \frac{u^{2}}{s}} e^{-c_{2} \frac{u^{2}}{t-s}} d u \leq \\
\int_{|u|>\frac{1}{\sqrt{\epsilon}}} e^{-c_{2} \frac{u^{2}}{t}} d u \leq \sqrt{\frac{t}{c_{2}}} \cdot \int_{|v|>\sqrt{\frac{c_{2}}{t}}} e^{-v^{2}} d v \leq \sqrt{\frac{t}{c_{2}}} \cdot e^{-\frac{c_{2}}{\epsilon t}} .
\end{gathered}
$$

The second integral involves volume of the manifold $\tilde{M}$. Modulo negligible error (up to a contribution from a compact set) we can look at it as the integral over the outside of the ball centered at the fixed point $\bar{y} \in \bar{M}$ with radius $R=1+\operatorname{diam} \bar{M}$. We do have

$$
\begin{gathered}
\int_{\{z ; d(z, \bar{y})>R\}} e^{-c_{2} \frac{d^{2}(z ; \bar{y}}{s}} e^{-c_{2} \frac{d^{2}(z ; \bar{y}}{t-s}} d z \leq \int_{\{z ; d(z, \bar{y})>R\}} e^{-c_{2} \frac{d^{2}(z ; \bar{y}}{t}} d z \leq \\
c_{3} \int_{R}^{\infty} e^{-c_{2} \frac{r^{2}}{t}} e^{c_{4} r} d r \leq c_{5} \int_{R}^{\infty} e^{-c_{6} \frac{r^{2}}{t}} d r \leq
\end{gathered}
$$


38I-T. BENAMEUR, A. L. CAREY, J. PHILliPS, A. RENNIE, F. A. SUKOCHEV, AND K. P. WOJCIECHOWSKI

$$
c_{5} \sqrt{\frac{t}{c_{6}}} \cdot \int_{v>\sqrt{\frac{c_{6}}{t}} R} e^{-v^{2}} d v \leq c_{5} \sqrt{\frac{t}{c_{6}}} \cdot e^{-c_{6} \frac{R^{2}}{t}}
$$

\section{Spectral Flow FOr almost periodic gauge transformations}

9.1. Shubin's framework. We follow Shubin 62, 63, which in turn extends the original paper of Coburn et al [24, 25]. In this paragraph, we review the definition of the von Neumann algebra which is appropriate for the study of almost periodic operators. Recall that a trigonometric function is a finite linear combination of exponential functions $e_{\xi}: x \mapsto e^{i<x, \xi>}$. The space $\operatorname{Trig}\left(\mathbf{R}^{n}\right)$ of trigonometric functions is clearly a $*$-subalgebra of the $C^{*}$-algebra $C_{b}\left(\mathbf{R}^{n}\right)$ of continuous bounded functions. The uniform closure of $\operatorname{Trig}\left(\mathbf{R}^{n}\right)$ is thus a $C^{*}$-algebra called the algebra of almost periodic functions and denoted $\mathcal{A} P\left(\mathbf{R}^{n}\right)$. Since this $C^{*}$-algebra is unital and commutative, it is the $C^{*}$-algebra of continuous functions on a compact space $\mathbf{R}_{B}^{n}$ which is a compactification of $\mathbf{R}^{n}$ with respect to the appropriate topology. The compact space $\mathbf{R}_{B}^{n}$ is called the Bohr compactification of $\mathbf{R}^{n}$ or simply the Bohr space. Addition extends to $\mathbf{R}_{B}^{n}$ which is a compact abelian group containing $\mathbf{R}^{n}$ as a dense subgroup. There is a unique normalized Haar measure $\alpha_{B}$ on $\mathbf{R}_{B}^{n}$ such that the family $\left(e_{\xi}\right)_{\xi \in \mathbf{R}^{n}}$ is orthonormal. Namely, the measure $\alpha_{B}$ is given for any almost periodic function $f$ on $\mathbf{R}^{n}$ by:

$$
\alpha_{B}(f):=\lim _{T \rightarrow+\infty} \frac{1}{(2 T)^{n}} \int_{(-T, T)^{n}} f(x) d x .
$$

By using the measure $\alpha_{B}$ one defines the Hilbert space completion $L^{2}\left(\mathbf{R}_{B}^{n}\right)$ of $\operatorname{Trig}\left(\mathbf{R}^{n}\right)$. This Hilbert space is called the Besicovich space and it has an orthonormal basis given by $\left(e_{\xi}\right)_{\xi \in \mathbf{R}^{n}}$. In other words, the Pontryagin dual of $\mathbf{R}_{B}^{n}$ is the discrete abelian group $\mathbf{R}_{d}^{n}$ and the Fourier transform $\mathcal{F}_{B}: \ell^{2}\left(\mathbf{R}_{d}^{n}\right) \longrightarrow L^{2}\left(\mathbf{R}_{B}^{n}\right)$ is given by:

$$
\mathcal{F}_{B}\left(\delta_{\xi}\right)=e_{\xi}, \quad \text { with } \delta_{\xi}(\eta)=\delta_{\xi, \eta},
$$

where $\delta_{\xi, \eta}$ is the Kronecker symbol. We shall denote by $\mathcal{F}$ the usual Fourier transform on the abelian group $\mathbf{R}^{n}$ with its usual Lebesgue measure.

For any $f \in C_{b}\left(\mathbf{R}^{n}\right)$ we shall denote, for any vector $\lambda \in \mathbf{R}^{n}$, by $T_{\lambda} f$ the translated function defined by $\left(T_{\lambda} f\right)(x)=f(x-\lambda)$. Let $f \in C_{b}\left(\mathbf{R}^{n}\right)$ and let $\epsilon>0$ be given. A vector $\lambda \in \mathbf{R}^{n}$ is called an $\epsilon$ - period for $f$ if the uniform norm of $T_{\lambda} f-f$ is bounded by $\epsilon$, i.e.

$$
\left\|T_{\lambda} f-f\right\|_{\infty}:=\sup _{t \in \mathbf{R}^{n}}|f(t-\lambda)-f(t)| \leq \epsilon .
$$

A subset $E$ of $\mathbf{R}^{n}$ is relatively dense if there exists $T>0$ such that

$$
\forall x \in \mathbf{R}^{n}, \exists u \in E: u-x \in\left[-\frac{T}{2},+\frac{T}{2}\right]^{n} .
$$

It is worth pointing out that, for any function $f: \mathbf{R}^{n} \rightarrow \mathbf{C}$, the following properties are equivalent 24]:

- $f$ is an almost periodic function. 
- $f$ is a continuous bounded function whose $\epsilon$ periods are relatively dense for every $\epsilon>0$.

It is clear from the second characterization of an almost periodic function that any periodic function is almost periodic. An interesting class of examples arises from the study of quasiperiodic functions. Assume for simplicity that $n=1$ and let $\alpha=\left(\alpha_{1}, \cdots, \alpha_{p}\right) \in \mathbf{R}^{p}$ be a fixed list of real numbers. Then for any summable sequence $c=\left(c_{m}\right)_{m \in \mathbf{Z}^{p}}$, we get an almost periodic function on $\mathbf{R}$ by setting:

$$
\varrho_{c, \alpha}(x):=\sum_{m \in \mathbf{Z}^{p}} c_{m} e^{2 i \pi<m, \alpha>x} .
$$

Then more complicated examples of almost periodic functions arise as limits of periodic or quasi-periodic functions. For instance, the function $\sum_{n \geq 0} a_{n} \cos \left(\frac{x}{2^{n}}\right)$ where $\sum_{n}\left|a_{n}\right|<+\infty$, is an almost periodic function.

The action of $\mathbf{R}^{n}$ on $\mathbf{R}_{B}^{n}$ by translations yields a topological dynamical system whose naturally associated von Neumann algebra is the crossed product von Neumann algebra $L^{\infty}\left(\mathbf{R}_{B}^{n}\right) \rtimes \mathbf{R}^{n}$. It is more convenient for applications to consider the commutant of this von Neumann algebra denoting it by $\mathcal{N}$. It is also a crossed product. This time it is the von Neumann algebra $L^{\infty}\left(\mathbf{R}^{n}\right) \rtimes \mathbf{R}_{d}^{n}$. The von Neumann algebra $\mathcal{N}$ is a type $\mathrm{II}_{\infty}$ factor with a faithful normal semifinite trace $\tau$. It can be described as the set of Borel essentially bounded families $\left(A_{\mu}\right)_{\mu \in \mathbf{R}_{B}^{n}}$ of bounded operators in $L^{2}\left(\mathbf{R}^{n}\right)$ which are $\mathbf{R}^{n}$-equivariant, i.e. such that

$$
A_{\mu}=\sigma_{\mu}\left(A_{0}\right)=T_{-\mu} A_{0} T_{\mu}, \quad \forall \mu \in \mathbf{R}^{n} .
$$

Here and in the sequel we denote by $\sigma_{\mu}$ conjugation of any operator with the translation $T_{\mu}$ so that $\sigma_{\mu}(B)=T_{-\mu} B T_{\mu}$. If we denote by $M_{\varphi}$ the operator of multiplication by a bounded function $\varphi$, then examples of such families are given for any $\lambda$ by the families

$$
\left(\sigma_{\mu}\left(M_{e_{\lambda}}\right)\right)_{\mu \in \mathbf{R}_{B}^{n}} .
$$

We choose the Fourier transform

$$
\mathcal{F} f(\zeta)=\frac{1}{(2 \pi)^{n / 2}} \int_{\mathbf{R}^{n}} e^{i x \zeta} f(x) d x .
$$

Now the von Neumann algebra $\mathcal{N}$ can be defined [24, 25] as the double commutant of the set of operators $\left\{M_{e_{\lambda}} \otimes M_{e_{\lambda}}, T_{\lambda} \otimes 1 \mid \lambda \in \mathbf{R}^{n}\right\}$ on the Hilbert space $\mathcal{H}=L^{2}\left(\mathbf{R}^{n}\right) \otimes L^{2}\left(\mathbf{R}_{B}^{n}\right)$

There is a natural way to imbed the $C^{*}$-algebra $\mathcal{A} P\left(\mathbf{R}^{n}\right)$ in $\mathcal{N}$ by setting

$$
\pi(f):=\left(\sigma_{\mu}\left(M_{f}\right)\right)_{\mu \in \mathbf{R}_{B}^{n}}
$$

This family then belongs to $\mathcal{N}$ and $\pi$ is clearly faithful. Viewed as an operator on $\mathcal{H}, \pi(f)$ is given by $\pi(f)(g)(x, \mu)=f(x+\mu) g(x, \mu)$. If $B=\left(B_{\mu}\right)_{\mu}$ is a positive element of $\mathcal{N}$, then we define the expectation $E(B)$ as the Haar integral:

$$
E(B):=\int_{\mathbf{R}_{B}^{n}} B_{\mu} d \alpha_{B}(\mu) .
$$

Since the family $B$ is translation equivariant and since $\alpha_{B}$ is translation invariant, the operator $E(B)$ clearly commutes with the translation in $L^{2}\left(\mathbf{R}^{n}\right)$ and is therefore given by a Fourier multiplier $\widetilde{M}\left(\varphi_{B}\right)$ with $\varphi_{B}$ a positive element of $L^{\infty}\left(\mathbf{R}^{n}\right)$. Recall that the Fourier multiplier 
$\widetilde{M}\left(\varphi_{B}\right)$ is conjugation of the multiplication operator $M_{\varphi}$ by the Fourier transform, i.e. $\widetilde{M}\left(\varphi_{B}\right)=$ $\mathcal{F}^{-1} M_{\varphi} \mathcal{F}$. When the function $\varphi$ is for instance in the Schwartz space, the operator $\widetilde{M}\left(\varphi_{B}\right)$ is convolution by the Schwartz function $\frac{1}{(2 \pi)^{n / 2}} \mathcal{F}^{-1} \varphi$. Hence the expectation $E$ takes values in the von Neumann algebra $\widetilde{M}\left(L^{\infty}\left(\mathbf{R}^{n}\right)\right)$, i.e.

$$
E: \mathcal{N} \longrightarrow \widetilde{M}\left(L^{\infty}\left(\mathbf{R}^{n}\right)\right)
$$

Now, using the usual Lebesgue integral on $\mathbf{R}^{n}$, we use the normalisation of Coburn et al [24] and introduce the following definition of the trace $\tau$ :

$$
\tau(B)=\int_{\mathbf{R}^{n}} \varphi_{B}(\zeta) d \zeta
$$

Lemma 9.1. 24, 25] The map $\tau$ is, up to constant, the unique positive normal faithful semifinite trace on $\mathcal{N}$.

The space $L^{1}(\mathcal{N}, \tau)$ of trace-class $\tau$-measurable operators with respect to $\mathcal{N}$ is the space of $\tau$-measurable operators $T$ as explained by Fack et al [38] such that $\int_{(0,+\infty)} \mu_{s}^{\tau}(T) d s<+\infty$. Here $\mu_{s}^{\tau}(T)$ is the $s$-th characteristic value of $T$ [38, for the precise definitions. More generally and for any $p \geq 1$, we shall denote by $L^{p}(\mathcal{N}, \tau)$ the space of $\tau$-measurable operators $T$ such that $\left(T^{*} T\right)^{p / 2} \in L^{1}(\mathcal{N}, \tau)$. It is well known that the space $L^{p}(\mathcal{N}, \tau) \cap \mathcal{N}$ is a two-sided $*$-ideal in $\mathcal{N}$ that we shall call the $p$-th Schatten ideal of $\mathcal{N}$.

We also consider the Dixmier space $L^{1, \infty}(\mathcal{N}, \tau)$ of those operators $T \in \mathcal{N}$ such that

$$
\int_{0}^{s} \mu_{t}^{\tau}(T) d t \sim O(\log (s)) .
$$

Again, $L^{1, \infty}(\mathcal{N}, \tau)$ is a two-sided $*$-ideal in $\mathcal{N}$. There are well defined Dixmier traces $\tau_{\omega}$ on $L^{1, \infty}(\mathcal{N}, \tau)$ parametrized by limiting processes $\omega[6,21$.

Consider the trace on the von Neumann algebra $\mathcal{N}$ evaluated on an operator of the form $M_{a} K$ where $a$ is almost periodic and $K$ is a convolution operator on $L^{2}\left(\mathbf{R}^{n}\right)$ arising from multiplication by an $L^{1}$ function $k$ on the Fourier transform. We have,

$$
\tau\left(M_{a} K\right)=\lim _{T \rightarrow+\infty} \frac{1}{(2 T)^{n}} \int_{(-T, T)^{n}} a(x) d x \int_{\mathbf{R}^{n}} k(\zeta) d \zeta
$$

More generally, any pseudodifferential operator $A$ on $L^{2}\left(R^{n}, \mathbf{C}^{N}\right)$ with almost periodic coefficients of nonpositive order $m$ acting on $\mathbf{C}^{N}$-valued functions, can be viewed as a family over $\mathbf{R}_{B}^{n}$ of pseudodifferential operators on $\mathbf{R}^{n}$. To do this first take the symbol $a$ of $A$, then the operator $\sigma_{\mu}(A)$ is the pseudodifferential operator with almost periodic coefficients whose symbol is

$$
(x, \xi) \longmapsto a(x+\mu, \xi) .
$$

When $m \leq 0$, we get in this way an element of the von Neumann algebra $\mathcal{N}$. We denote by $\Psi_{A P}^{0}$ the algebra of pseudodifferential operators with almost periodic coefficients and with non positive order. When the order $m$ of $A$ is $>0$ then the operator $A^{\sharp}$ given by the family $\left(\sigma_{\mu}(A)\right)_{\mu \in \mathbf{R}_{B}^{n}}$ is affiliated with the von Neumann algebra $\mathcal{N}$. If the order $m$ of $A$ is $<-n$, then 
the bounded operator $A^{\sharp}$ is trace class with respect to the trace $\tau$ on the von Neumann algebra $\mathcal{N} \otimes M_{N}(\mathbf{C})[64][$ Proposition 3.3] and we have:

$$
\tau\left(A^{\sharp}\right)=\lim _{T \rightarrow+\infty} \frac{1}{(2 T)^{n}} \int_{(-T,+T)^{n} \times \mathbf{R}^{n}} \operatorname{tr}(a(x, \zeta)) d x d \zeta
$$

Indeed, the expectation $E\left(A^{\sharp}\right)$ is a pseudodifferential operator on $\mathbf{R}^{n}$ with symbol denoted by $E(a)$ and is independent of the $x$-variable, it is given by:

$$
E(a)(\zeta)=\lim _{T \rightarrow+\infty} \frac{1}{(2 T)^{n}} \int_{(-T,+T)^{n}} a(x, \zeta) d x .
$$

Hence the operator $E\left(A^{\sharp}\right)$ is precisely the Fourier multiplier $\widetilde{M}(E(a))$ and so:

$$
\tau\left(A^{\sharp}\right)=\int_{\mathbf{R}^{n}} \operatorname{tr}(E(a)(\zeta)) d \zeta .
$$

Let $\Psi_{A P}^{\infty}$ be the space of one step polyhomogeneous classical pseudodifferential operators on $\mathbf{R}^{n}$ with almost periodic coefficients.

Theorem 9.2. Let $A$ be a (scalar) pseudodifferential operator with almost periodic coefficients on $\mathbf{R}^{n}$. We assume that the order $m$ of $A$ is $\leq-n$ and we denote by $a_{-n}$ the $-n$ homogeneous part of the symbol $a$. Then the operator $A^{\sharp}$ belongs to the Dixmier ideal $L^{1, \infty}(\mathcal{N}, \tau)$. Moreover, the Dixmier trace $\tau_{\omega}\left(A^{\sharp}\right)$ of $A^{\sharp}$ associated with a limiting process $\omega$ does not depend on $\omega$ and is given by the formula:

$$
\tau_{\omega}\left(A^{\sharp}\right)=\frac{1}{n} \int_{\mathbf{R}_{B}^{n} \times \mathbf{S}^{n-1}} a_{-n}(x, \zeta) d \alpha_{B}(x) d \zeta .
$$

Proof. We denote as usual by $\Delta$ the Laplace operator on $\mathbf{R}^{n}$. The operator $A(1+\Delta)^{n / 2}$ is then a pseudodifferential operator with almost periodic coefficients and nonpositive order. Hence, the operator $\left[A(1+\Delta)^{n / 2}\right]^{\sharp}=A^{\sharp}\left(1+\Delta^{\sharp}\right)^{n / 2}$ belongs to the von Neumann algebra $\mathcal{N}$. Now the operator $\left(1+\Delta^{\sharp}\right)^{-n / 2}$ is a Fourier multiplier defined by the function $\zeta \mapsto\left(1+\zeta^{2}\right)^{n / 2}$. Hence if, for $\lambda>0, E_{\lambda}$ is the spectral projection of the operator $(1+\Delta)^{-n / 2}$ corresponding to the interval $(0, \lambda)$ then the operator $1-E_{\lambda}$ is the Fourier multiplier defined by the function $\left.\zeta \mapsto 1_{(\lambda,+\infty)}\left(\zeta^{2}+1\right)^{n / 2}\right)$. It follows that the trace $\tau$ of the operator $1-E_{\lambda}$ is given by

$$
\int_{\mathbf{R}^{n}} 1_{(\lambda,+\infty)}\left(\frac{1}{\left(\zeta^{2}+1\right)^{n / 2}}\right) d \zeta
$$

It is easy to compute this integral and to show that it is proportional to $\frac{1}{\lambda}$. So the infimum of those $\lambda$ for which $\tau\left(1-E_{\lambda}\right) \leq t$ is precisely proportional to $\frac{1}{t}$. Hence the operator $\left(1+\Delta^{\sharp}\right)^{-n / 2}$, and hence $A$, belongs to the Dixmier ideal $L^{1, \infty}(\mathcal{N}, \tau)$.

In order to compute the Dixmier trace of the operator $A$, we apply Shubin 62] [Theorem 10.1] to deduce that the spectral $\tau$-density $N_{A}(\lambda)$ of $A$ has the asymptotic expansion

$$
N_{A}(\lambda)=\frac{\chi_{0}(A)}{\lambda}(1+o(1)), \quad \lambda \rightarrow+\infty,
$$


where $\chi_{0}(A)$ is given by:

$$
\chi_{0}(A)=\frac{1}{n} \int_{\mathbf{R}_{B}^{n} \times \mathbf{S}^{n-1}} a_{-n}(x, \zeta) d \alpha_{B}(x) d \zeta .
$$

Now, if $A$ is positive then by Benameur et al [6] [Proposition 1]:

$$
\tau_{\omega}(A)=\lim _{\lambda \rightarrow+\infty} \lambda N_{A}(\lambda)=\chi_{0}(A) .
$$

This proves the theorem for positive $A$. Since the principal symbol map is a homomorphism, we deduce the result for general $A$.

The reader familiar with the Wodzicki residue will observe that the normalisation we have chosen for the trace in the von Neumann setting of this Section eliminates a factor of $\frac{1}{(2 \pi)^{n}}$ which occurs at the corresponding point in the type I theory.

\section{THE ODD SEMIFINITE LOCAL INDEX THEOREM}

The original type I version of this result is due to Connes-Moscovici 32. There are two new proofs, one due to Higson [43] and one due to Carey et al [18. The latter argument handles the case of semifinite spectral triples. Quite remarkably this very general odd semifinite local index theorem is proved by starting from the integral formulae for spectral flow that we have described in earlier sections. We do not have the space here to explain how it is done.

We restrict our discussion to a statement of the theorem. First, we require multi-indices $\left(k_{1}, \ldots, k_{m}\right), k_{i} \in\{0,1,2, \ldots\}$, whose length $m$ will always be clear from the context. We write $|k|=k_{1}+\cdots+k_{m}$, and define $\alpha(k)$ by

$$
\alpha(k)=1 / k_{1} ! k_{2} ! \cdots k_{m} !\left(k_{1}+1\right)\left(k_{1}+k_{2}+2\right) \cdots(|k|+m) .
$$

The numbers $\sigma_{n, j}$ are defined by the equality

$$
\prod_{j=0}^{n-1}(z+j+1 / 2)=\sum_{j=0}^{n} z^{j} \sigma_{n, j}
$$

with $\sigma_{0,0}=1$. These are just the elementary symmetric functions of $1 / 2,3 / 2, \ldots, n-1 / 2$.

If $(\mathcal{A}, \mathcal{H}, \mathcal{D})$ is a smooth semifinite spectral triple (ie $\mathcal{A}$ is in the domain of $\delta^{n}$ for all $n$ where $\left.\delta(a)=\left[\left(1+D^{2}\right)^{1 / 2}, a\right]\right)$ and $T \in \mathcal{N}$, we write $T^{(n)}$ to denote the iterated commutator

$$
\left[\mathcal{D}^{2},\left[\mathcal{D}^{2},\left[\cdots,\left[\mathcal{D}^{2}, T\right] \cdots\right]\right]\right]
$$

where we have $n$ commutators with $\mathcal{D}^{2}$. It follows 18 that operators of the form

$$
T_{1}^{\left(n_{1}\right)} \cdots T_{k}^{\left(n_{k}\right)}\left(1+\mathcal{D}^{2}\right)^{-\left(n_{1}+\cdots+n_{k}\right) / 2}
$$

are in $\mathcal{N}$ when $T_{i}=\left[\mathcal{D}, a_{i}\right]$, or $=a_{i}$ for $a_{i} \in \mathcal{A}$.

Definition 10.1. If $(\mathcal{A}, \mathcal{H}, \mathcal{D})$ is a smooth semifinite spectral triple, we call

$$
p=\inf \left\{k \in \mathbf{R}: \tau\left(\left(1+\mathcal{D}^{2}\right)^{-k / 2}\right)<\infty\right\}
$$


the spectral dimension of $(\mathcal{A}, \mathcal{H}, \mathcal{D})$. We say that $(\mathcal{A}, \mathcal{H}, \mathcal{D})$ has isolated spectral dimension if for $b$ of the form

$$
b=a_{0}\left[\mathcal{D}, a_{1}\right]^{\left(k_{1}\right)} \cdots\left[\mathcal{D}, a_{m}\right]^{\left(k_{m}\right)}\left(1+\mathcal{D}^{2}\right)^{-m / 2-|k|}
$$

the zeta functions

$$
\zeta_{b}(z-(1-p) / 2)=\tau\left(b\left(1+\mathcal{D}^{2}\right)^{-z+(1-p) / 2}\right)
$$

have analytic continuations to a deleted neighbourhood of $z=(1-p) / 2$.

Now we define, for $(\mathcal{A}, \mathcal{H}, \mathcal{D})$ having isolated spectral dimension and

$$
\begin{aligned}
b & =a_{0}\left[\mathcal{D}, a_{1}\right]^{\left(k_{1}\right)} \cdots\left[\mathcal{D}, a_{m}\right]^{\left(k_{m}\right)}\left(1+\mathcal{D}^{2}\right)^{-m / 2-|k|} \\
\tau_{j}(b) & =\operatorname{res}_{z=(1-p) / 2}(z-(1-p) / 2)^{j} \zeta_{b}(z-(1-p) / 2) .
\end{aligned}
$$

The hypothesis of isolated spectral dimension is clearly necessary here in order to define the residues. The semifinite local index theorem is as follows.

Theorem 10.2. Let $(\mathcal{A}, \mathcal{H}, \mathcal{D})$ be an odd finitely summable smooth spectral triple with spectral dimension $p \geq 1$. Let $N=[p / 2]+1$ where [.] denotes the integer part (so $2 N-1$ is the largest odd integer $\leq p+1)$, and let $u \in \mathcal{A}$ be unitary. Then if $(\mathcal{A}, \mathcal{H}, \mathcal{D})$ also has isolated spectral dimension then

$$
s f\left(\mathcal{D}, u^{*} \mathcal{D} u\right)=\frac{1}{\sqrt{2 \pi i}} \sum_{m}(-1)^{(m-1) / 2}\left(\frac{(m-1)}{2}\right) ! \phi_{m}\left(u, u^{*}, \ldots, u, u^{*}\right)
$$

where $\phi_{m}\left(u, u^{*}, \ldots, u, u^{*}\right)$ is

$$
\begin{gathered}
\sum_{|k|=0}^{2 N-1-m} \sum_{j=0}^{|k|+(m-1) / 2}(-1)^{|k|} \alpha(k) \sigma_{(|k|+(m-1) / 2), j} \\
\times \tau_{j}\left(u\left[\mathcal{D}, u^{*}\right]^{\left(k_{1}\right)} \cdots[\mathcal{D}, u]^{\left(k_{m}\right)}\left(1+\mathcal{D}^{2}\right)^{-|k|-m / 2}\right),
\end{gathered}
$$

When $[p]=2 n$ is even, the term with $m=2 N-1$ is zero, and for $m=1,3, \ldots, 2 N-3$, all the top terms with $|k|=2 N-1-m$ are zero.

We aim to compute the terms in this formula for semifinite spectral flow in the case where $\mathcal{D}$ is the Euclidean Dirac operator on the spin bundle $\mathcal{S}$ over $\mathbf{R}^{n}$ tensored with the trivial bundle rank $N$ bundle and $u$ is a smooth almost periodic function from $\mathbf{R}^{n}$ to $U(N)$.

\section{Almost Periodic speCtral triple}

We now apply the local index theorem to compute spectral flow. We thus assume that $n$ is odd. The von Neumann algebra constructed previously is non-separable and so to avoid a discussion of the non-separable situation we need to slightly modify our approach in this Section. In fact it is sufficient to study the dense countable abelian subgroups of $\mathbf{R}^{n}$. Let us fix one such, call it $D$ and explain how the theory works for this case. Consider the subalgebra $\mathcal{A}$ of $\mathcal{A} P\left(\mathbf{R}^{n}\right)$ consisting of almost periodic functions generated by $e_{\xi}$ with $\xi \in D$. We denote by $\mathcal{A}^{\infty}$ the $*$-subalgebra of $\mathcal{A} P\left(\mathbf{R}^{n}\right)$ consisting of functions in $\mathcal{A}$ which have bounded derivatives 
of all orders. The von Neumann algebra we now consider is the crossed product algebra of $D$ with $L^{\infty}\left(\mathbf{R}^{n}\right)$ )and is denoted by $\mathcal{M}$. We take the Hilbert space on which this algebra acts to be $B_{D}^{2}\left(\mathbf{R}^{n}\right) \otimes L^{2}\left(\mathbf{R}^{n}\right)$ where $B_{D}^{2}\left(\mathbf{R}^{n}\right)$ is the Hilbert space completion of $\mathcal{A}$ where the norm and inner product are given by the restriction of the Haar trace on $\mathcal{A} P^{\infty}\left(\mathbf{R}^{n}\right)$ to $\mathcal{A}$ (note that $\left.B_{D}^{2}\left(\mathbf{R}^{n}\right) \cong \ell^{2}(D)\right)$. This type $\mathrm{II}_{\infty}$ von Neumann algebra is endowed with a faithful normal semi-finite trace that we denote by $\tau$. (We note that the explicit formula for $\tau$ is as given in Section 9.)

The usual Dirac operator on $\mathbf{R}^{n}$ is denoted by $\partial$. So, if $\mathcal{S}$ carries the spin representation of the Clifford algebra of $\mathbf{R}^{n}$ then $\partial$ acts on smooth $\mathcal{S}$-valued functions on $\mathbf{R}^{n}$. The operator $\partial$ is $\mathbf{Z}^{n}$-periodic and it is affiliated with the von Neumann algebra $\mathcal{M}_{\mathcal{S}}=\mathcal{M} \otimes \operatorname{End}(\mathcal{S})$. This latter is also a type $\mathrm{II}_{\infty}$ von Neumann algebra with the trace $\tau \otimes \operatorname{tr}$. More generally, for any $N \geq 1$, we shall denote by $\mathcal{M}_{\mathcal{S}, N}$ the von Neumann algebra $\mathcal{M} \otimes \operatorname{End}\left(\mathcal{S} \otimes \mathbf{C}^{N}\right)$ with the trace $\tau \otimes \operatorname{tr}$.

The algebra $\mathcal{A}$ and its closure are faithfully represented as $*$-subalgebras of the von Neumann algebra $\mathcal{M}_{\mathcal{S}}$. In the same way the algebra $\mathcal{A} \otimes M_{N}(\mathbf{C})$ can be viewed as a $*$-subalgebra of $\mathcal{M}_{\mathcal{S}, N}$. More precisely, if $a \in \mathcal{A}$ then the operator $a^{\sharp}$ defined by:

$$
\left(a^{\sharp} f\right)(x, y):=a(x+y) f(x, y), \quad \forall f \in B_{D}^{2}\left(\mathbf{R}^{n}\right) \otimes L^{2}\left(\mathbf{R}^{n}\right),
$$

belongs to $\mathcal{M}$. The operator $a^{\sharp}$ is just the one associated with the zero-th order differential operator corresponding to multiplication by $a$. The same formula allows to represent $\mathcal{A} \otimes M_{N}(\mathbf{C})$ in $\mathcal{M}_{\mathcal{S}, N}$. For notional simplicity we put $N=1$ in the next result although we will use a general $N \geq 1$ in the subsequent subsection.

Proposition 11.1. The triple $\left(\mathcal{A}, \mathcal{M}_{\mathcal{S}}, \partial^{\sharp}\right)$ is a semifinite spectral triple of finite dimension equal to $n$.

Proof. Note that the algebra $\mathcal{A}$ is unital. The differential operator $\widetilde{\partial}$ is known to be densely defined, elliptic, periodic and self-adjoint on $L^{2}\left(\mathbf{R}^{n}, \mathcal{S}\right)$. Therefore, the operator $\widehat{\partial}^{\sharp}$ is affiliated with the von Neumann algebra $\mathcal{M}_{\mathcal{S}}$ and it becomes self-adjoint as a densely defined unbounded operator on the Hilbert space $B_{D}^{2}\left(\mathbf{R}^{n}\right) \otimes L^{2}\left(\mathbf{R}^{n}, \mathcal{S}\right)$ with $\check{\partial}^{2}=\Delta I$ where $I$ is the identity operator and $\Delta$ is the usual Laplacian. For any smooth bounded almost periodic function $f$ on $\mathbf{R}^{n}$, with bounded derivatives of all orders, the commutator $[ð, f]$ is a 0 -th order almost periodic differential operator and so $\left[\widetilde{\partial}^{\sharp}, f\right]$ belongs to the von Neumann algebra $\mathcal{M}_{\mathcal{S}}$.

On the other hand, the pseudodifferential operator $T=\left(\varpi^{2}+I\right)^{-1 / 2}$ is essentially the Fourier multiplier associated with the function $k \mapsto \frac{1}{\left(\|k\|^{2}+1\right)^{1 / 2}}$. Therefore, its singular numbers $\mu_{t}(T)$ can be computed explicitly as in the proof of Theorem 9.2 and shown to be proportional to $t^{-1 / n}$.

11.1. Analysis of terms in the above example. First we note that the spectral dimension is the dimension $n$ of the underlying Euclidean space and this is assumed to be odd. It follows that the summation over $|k|$ in each term in the preceding theorem is over the range $0 \leq|k| \leq n-m$. Second we note that $m$ is always odd. 
Let us write $e_{1}, e_{2}, \ldots, e_{n}$ for an orthonormal basis of $\mathbf{R}^{n}, c\left(e_{1}\right), c\left(e_{2}\right), \ldots, c\left(e_{n}\right)$ for the corresponding Clifford generators. So we have $c\left(e_{i}\right) c\left(e_{j}\right)+c\left(e_{j}\right) c\left(e_{i}\right)=2 \delta_{i j} 1$ and we can write $\widetilde{\partial}=\sum i c\left(e_{j}\right) \otimes 1 \partial_{j}$ where 1 just denotes the identity matrix. We let $u \in \mathcal{A}^{\infty} \otimes \operatorname{End}\left(\mathbf{C}^{N}\right)$ be unitary. Thus $u\left[\widetilde{\partial}, u^{*}\right]=\sum i c\left(e_{j}\right) \otimes \partial_{j} u^{*}$ The trace is now the product of the trace on the spinor part times the von Neumann trace composed with the matrix trace on the matrices acting on $V$. This very simple structure enables us to eliminate all but one of the terms in the local index formula by first taking the trace of the product of Clifford generators. Note that the trace on the Clifford algebra in the spin representation is given by

$$
\operatorname{Tr}_{\text {Spin }}\left(i^{n} c\left(e_{1}\right) c\left(e_{2}\right) \ldots c\left(e_{n}\right)\right)=i^{-[(n+1) / 2]} 2^{(n-1) / 2}
$$

and the trace on any product of $0<k<n$ generators is zero.

A typical term in the local index formula is proportional to

$$
\tau_{j}\left(u\left[ð, u^{*}\right]^{\left(k_{1}\right)}[ð, u]^{\left(k_{2}\right)} \ldots[\widetilde{\partial}, u]^{\left(k_{m-1}\right)}\left[ð, u^{*}\right]^{\left(k_{m}\right)}(1+\Delta)^{-|k|-m / 2}\right)
$$

This is, up to a sign, a product of factors of the form $\left(u ð u^{*}-\not \partial\right)^{\left(k_{l}\right)}$. The Laplacian commutes with $\partial$ so that a typical factor is of the form $\sum_{i} c\left(e_{i}\right) \otimes g_{i}$ and the $g_{i}$ are $N \otimes N$ matrix valued pseudodifferential operators. Since there is always a product of an odd number of factors of this form $\sum_{j} c\left(e_{j}\right) \otimes g_{j}$ in a term $(*)$ the trace on the Clifford elements will produce zero unless $m=n$. In that case $|k|$ is forced to be zero.

Thus only one term survives in the local index theorem and that term is (see appendix)

$$
\frac{(-1)^{(n-1) / 2}}{n 2^{(n-1)}} \tau_{0}\left(u\left[\text { ð, } u^{*}\right][ð, u]\left[ð, u^{*}\right][ð, u] \ldots[ð, u]\left[ð, u^{*}\right](1+\Delta)^{-n / 2}\right)
$$

To compute this we first take care of the Clifford algebra. Using the fact that $[ð, u]=-u\left[ð, u^{*}\right] u$ we write the formula for the spectral flow as

$$
\frac{(-1)^{n}}{n 2^{(n-1)}} \tau_{0}\left(\left([ð, u] u^{*}\right)^{n}(1+\Delta)^{-n / 2}\right)
$$

We let $\operatorname{Tr}_{N}$ be the matrix trace on the auxiliary vector space. Now

$$
\left([ð, u] u^{*}\right)=\sum i \partial_{j}(u) u^{*} c\left(d x_{j}\right) .
$$

Writing $f_{j}=\partial_{j}(u) u^{*}$ we then have

$$
\left([\mathcal{D}, u] u^{*}\right)^{n}=i^{n} \sum_{J=\left(j_{1}, \ldots, j_{n}\right)} f_{j_{1}} \cdots f_{j_{n}} c\left(e_{j_{1}}\right) \cdots c\left(e_{j_{n}}\right),
$$

where the sum is extended over all multi-indices $J$. Every term in the sum is a multiple of the volume form, and so has non-zero (spinor) trace. In terms of permutations we have

$$
\begin{gathered}
\left([\mathcal{D}, u] u^{*}\right)^{n}=i^{n}\left(\sum_{\sigma \in \Sigma^{n}}(-1)^{\sigma} f_{\sigma(1)} \cdots f_{\sigma(n)}\right) c\left(e_{1}\right) \cdots c\left(e_{n}\right) \\
=: \Omega i^{n} c\left(e_{1}\right) \cdots c\left(e_{n}\right) .
\end{gathered}
$$


4M-T. BENAmEUR, A. L. CAREY, J. PHILliPS, A. RENNIE, F. A. SUKOCHEV, AND K. P. WOJCIECHOWSKI

In taking the trace we may first take the matrix trace over the Clifford endomorphisms of the spin bundle (with [...] denoting 'the integer part of') and so, with $\tau_{S}=\tau \times \operatorname{Tr}_{N} \times \operatorname{Tr}_{\text {Spin }}$

$$
\begin{aligned}
& \tau_{0}\left(\left([\mathcal{D}, u] u^{*}\right)^{n}\left(1+\mathcal{D}^{2}\right)^{-n / 2}\right)=\operatorname{res}_{s=0} \tau_{S}\left(\left([\mathcal{D}, u] u^{*}\right)^{n}\left(1+\mathcal{D}^{2}\right)^{-n / 2-s}\right) \\
& =r e s_{s=0} 2^{(n-1) / 2} i^{-[(n+1) / 2]}\left(\tau \times \operatorname{Tr}_{N}\right)\left(\Omega\left(1+\mathcal{D}^{2}\right)^{-n / 2-s}\right) \\
& =r e s_{s=0} \frac{2^{(n-1) / 2}}{i^{[(n+1) / 2]}} \lim \frac{1}{(2 T)^{n}} \int_{[-T, T]^{n}} \operatorname{Tr}_{N}(\Omega) \int_{\mathbf{R}^{n}}\left(1+|\xi|^{2}\right)^{-n / 2-s} d \xi \\
& =r e s \frac{2^{(n-1) / 2} V o l\left(S^{n-1}\right)}{i^{[(n+1) / 2]}} \lim \frac{1}{(2 T)^{n}} \int_{[-T, T]^{n}} \operatorname{Tr}_{N}(\Omega) \int_{0}^{\infty} \frac{r^{n-1}}{\left(1+r^{2}\right)^{n / 2+s}} d r \\
& =r e s_{s=0} \frac{2^{(n-1) / 2} V o l\left(S^{n-1}\right)}{i[(n+1) / 2]} \lim \frac{1}{(2 T)^{n}} \int_{[-T, T]^{n}} \operatorname{Tr}_{N}(\Omega) \frac{\Gamma(n / 2) \Gamma(s)}{2 \Gamma(n / 2+s)} \\
& =\frac{2^{(n-1) / 2}}{i^{(n+1) / 2}} \operatorname{Vol}\left(S^{n-1}\right) \frac{1}{2} \lim \frac{1}{(2 T)^{n}} \int_{[-T, T]^{n}} \operatorname{Tr}_{N}(\Omega) .
\end{aligned}
$$

Now

$$
\operatorname{Vol}\left(S^{n-1}\right)=\frac{(4 \pi)^{n / 2}}{2^{n-1} \Gamma(n / 2)} .
$$

Putting the previous calculations together gives our final result.

Theorem 11.2. With the notation as above the spectral flow along any path joining the Dirac operator $\partial$ to its gauge equivalent transform $u ð u^{*}$ by an almost periodic $U(N)$ valued function on $\mathbf{R}^{n}$ is given by the following formula:

$$
s f\left(ð, u \gtrsim u^{*}\right)=\frac{-i^{-[(n+1) / 2]} \pi^{n / 2}}{\Gamma(1+n / 2) 2^{(n+1) / 2}} \lim _{T \rightarrow \infty} \frac{1}{(2 T)^{n}} \int_{(-T, T)^{n}} t_{N}(\Omega)
$$

\section{APPENDIX}

12.1. Coefficients from the Local Index Theorem. The formula provided by the local index theorem for the special case considered in Section 11 is

$$
s f\left(\mathcal{D}, u^{*} \mathcal{D} u\right)=\frac{1}{\sqrt{2 \pi i}} \sum_{m=1}^{n}(-1)^{(m-1) / 2}\left(\frac{(m-1)}{2}\right) ! \phi_{m}\left(u, u^{*}, \ldots, u, u^{*}\right)
$$

where $\phi_{m}\left(u, u^{*}, \ldots, u, u^{*}\right)$ is given in Theorem 10.2. We already know that we need only compute the top term (degree $n$ ) of the local index theorem, because the Clifford trace will kill all the other terms. Since we have a simple spectral triple, the only multi-index $k=\left(k_{1}, \ldots, k_{n}\right)$ which arises is zero. In particular, we require $\alpha(0)=\frac{1}{n !}$. 
The numbers $\sigma_{m, j}$ are defined by the equality

$$
\prod_{l=0}^{m-1}(z+l+1 / 2)=\sum_{j=0}^{m} z^{j} \sigma_{m, j}
$$

These are just the elementary symmetric functions of $1 / 2,3 / 2, \ldots, m-1 / 2$. When $m=0$, this is the empty product, so $\sigma_{0,0}=1$. For $|k|=0$ we have $h:=|k|+(n-1) / 2=(n-1) / 2$ and because we have simple dimension spectrum, we only want $j=0$. Then $\sigma_{(n-1) / 2,0}$ is the coefficient of 1 in the product $\prod_{l=0}^{(n-3) / 2}(z+l+1 / 2)$. This is the product of all the non- $z$ terms, which is

$$
(1 / 2)(3 / 2) \times \cdots \times((n-3) / 2+1 / 2)=\frac{1.3 \cdot \cdots \cdot(2(n-1) / 2-1)}{2^{(n-1) / 2}} .
$$

The reason for writing this so elaborately, is that in this form it is obvious that it is equal to

$$
\frac{1}{\sqrt{\pi}} \Gamma((n-1) / 2+1 / 2)=\frac{1}{\sqrt{\pi}} \Gamma(n / 2) .
$$

Combining all these calculations gives us

$$
\phi_{n}\left(a_{0}, a_{1}, \ldots, a_{n}\right)=\sqrt{2 \pi i} \frac{\Gamma(n / 2)}{\sqrt{\pi} n !} \tau_{0}\left(a_{0}\left[\mathcal{D}, a_{1}\right] \cdots\left[\mathcal{D}, a_{n}\right]\left(1+\mathcal{D}^{2}\right)^{-n / 2}\right) .
$$

12.2. Constants from $C h_{n}\left(u^{*}\right)$ and pairing. When we pair $\phi_{n}$ with the Chern character of a unitary, we divide out by $\sqrt{2 \pi i}$, which is only in the Chern character of $(\mathcal{A}, \mathcal{H}, \mathcal{D})$ to make it compatible with the Kasparov product. The Chern character of $u^{*}$ has degree $n$ component

$$
\left.(-1)^{(n-1) / 2}((n-1) / 2)\right) ! u \otimes u^{*} \otimes u \otimes \cdots \otimes u^{*} \in \mathcal{A}^{\otimes n+1} .
$$

So $\left(\right.$ since $\left.s f\left(\mathcal{D}, u \mathcal{D} u^{*}\right)=\frac{1}{\sqrt{2 \pi i}} \phi_{n}\left(C h_{n}\left(u^{*}\right)\right)\right)$

$$
\begin{gathered}
s f\left(\mathcal{D}, u \mathcal{D} u^{*}\right)=\frac{(-1)^{(n-1) / 2} \Gamma(n / 2) \Gamma((n+1) / 2)}{\sqrt{\pi} n !} \\
\times \tau_{0}\left(u\left[\mathcal{D}, u^{*}\right] \cdots\left[\mathcal{D}, u^{*}\right]\left(1+\mathcal{D}^{2}\right)^{-n / 2}\right)
\end{gathered}
$$

Using the duplication formula for the Gamma function, we can simplify the constant in the last displayed formula. The duplication formula yields

$$
\Gamma(n / 2) \Gamma(n / 2+1 / 2)=\sqrt{\pi} \Gamma(n) 2^{-n+1}=\sqrt{\pi}(n-1) ! 2^{-n+1},
$$

and inserting this gives

$$
s f\left(\mathcal{D}, u \mathcal{D} u^{*}\right)=\frac{(-1)^{(n-1) / 2}}{n 2^{(n-1)}} \tau_{0}\left(u\left[\mathcal{D}, u^{*}\right] \cdots\left[\mathcal{D}, u^{*}\right]\left(1+\mathcal{D}^{2}\right)^{-n / 2}\right) .
$$




\section{REFERENCES}

[1] M. F. Atiyah, Elliptic operators, discrete groups and von Neumann algebras, Asterisque, 32-32(1976),4372 .

[2] M. F. Atiyah, V. Patodi, and I. M. Singer, Spectral asymmetry and Riemannian geometry, I, Proc. Camb. Phil. Soc., 77(1975), 43-69.

[3] M. F. Atiyah, V. Patodi, and I. M. Singer, Spectral asymmetry and Riemannian geometry. III, Proc. Camb. Phil. Soc., 79(1976), 71-99.

[4] M. F. Atiyah and I. M. Singer, Index theory for skew-adjoint Fredholm operators, Publ. Math. Inst. Hautes Etudes Sci., series 37(1969), 5-26.

[5] J. Avron, R. Seiler and B. Simon, The index of a pair of projections, J. Funct. Anal. 120 (1994), $220-237$.

[6] M-T. Benameur and T. Fack Type II Noncommutative geometry, I. Dixmier trace in von Neumann algebras, Adv. Math, (to appear). ¿iDEFANGED.175 ¿iDEFANGED.16

[7] J-M. Bismut and D. S. Freed The analysis of elliptic families II. Dirac operators, eta invariants and the holonomy theorem, Commun. Math. Phys. 107 (1986) 103-163.

[8] B. Booß-Bavnbek and K. Furutani, The Maslov index: a functional analytic definition and the spectral flow formula, Tokyo J. Math., 21,No. 1(1998), 1-34.

[9] B. Booß-Bavnbek, M. Lesch and J. Phillips Unbounded Fredholm operators and spectral flow, Canad. J. Math. 57 (2005) 225-250.

[10] B. Booß-Bavnbek and K. P. Wojciechowski, Elliptic boundary problems for Dirac operators, Birkhäuser, Berlin, 1993.

[11] M. Breuer, Fredholm theories in von Neumann algebras. I, Math. Ann., 178(1968), 243-254.

[12] M. Breuer, Fredholm theories in von Neumann algebras. II, Math. Ann., 180(1969), 313-325.

[13] L. G. Brown, R. G. Douglas and P. A. Fillmore, Extensions of $C^{*}$-algebras and K-homology, Ann. Math 105(1977), 349-363.

[14] A. L. Carey and J. Phillips, Algebras almost commuting with Clifford algebras in a $I I_{\infty}$ factor, $K$ Theory, 4(1991), 445-478.

[15] A. L. Carey and J. Phillips, Unbounded Fredholm modules and spectral flow, Canad J. Math., vol. 50 (1998), 673-718.

[16] A. L. Carey and J. Phillips, Spectral flow in $\Theta$-summable Fredholm modules, eta invariants and the JLO cocycle, K Theory 31 (2004) 135-194.

[17] A. L. Carey, J. Phillips, A. Rennie and F. A. Sukochev, The Hochschild class of the Chern character of semifinite spectral triples, J. Funct. Anal. 213 (2004) 111-153.

[18] A. L. Carey, J. Phillips, A. Rennie and F. A. Sukochev, The local index theorem in semifinite von Neumann algebras I. Spectral flow, Adv. Math, to appear.

[19] A. L. Carey, J. Phillips, A. Rennie and F. A. Sukochev, The local index theorem in semifinite von Neumann algebras II: the even case, Adv. Math, to appear.

[20] A. L. Carey, J. Phillips and F. A. Sukochev, On unbounded p-summable Fredholm modules, Adv. Math. 151 (2000) 140-163.

[21] A. L. Carey, J. Phillips and F. A. Sukochev, Spectral flow and Dixmier traces, Adv. Math. 173 (2003), 68-113.

[22] J. Cheeger, $\eta$-invariants, the adiabatic approximation and conical singularities, J. Diff. Geom. 26, (1987) $175-221$.

[23] J. Cheeger and M. Gromov, Bounds on the von Neumann dimension of $L^{2}$-cohomology and the GaussBonnet theorem for open manifolds, J. Diff. Geom. 21 (1985) 1-34.

[24] L. A. Coburn, R. G. Douglas, D. G. Schaeffer and I. M. Singer, $C^{*}$-algebras of operators on a half space II index theory, Publ. Math. Inst. Hautes Études Sci.,40 (1971) 69-80. 
[25] L. A. Coburn, R. D. Moyer and I. M. Singer, $C^{*}$-algebras of almost periodic pseudo-differential operators, Acta Math. 130 (1973), 279-307.

[26] A. Connes, Noncommutative differential geometry, Publ. Math. Inst. Hautes Études Sci., series 62 (1985), 41-44.

[27] A. Connes, Cyclic cohomology of Banach algebras and characters of $\theta$-summable Fredholm modules, $\mathrm{K}$ Theory 1(1988), 519-548.

[28] A. Connes, Compact metric spaces, Fredholm modules and hyperfiniteness, Ergodic Theory Dynam. Systems 9(1989), 207-220.

[29] A. Connes, Noncommutative Geometry, Acad. Press, San Diego, 1994.

[30] A. Connes and H. Moscovici, Cyclic cohomology, the Novikov conjecture and hyperbolic groups, Topology series 29 (1990), 345-388. ¿iDEFANGED.176 i.iDEFANGED.17

[31] A. Connes, Geometry from the spectral point of view, Lett. Math. Phys., 34 (1995) 203-238.

[32] A. Connes and H. Moscovici, The local index formula in noncommutative geometry, Geom. Funct. Anal. 5 (1995) 174-243.

[33] X. Dai and W. Zhang, Higher spectral flow, J. Funct. Anal. 157 (1998) 432-469.

[34] J. Dixmier, Les algèbres d'opérateurs dans l'espace Hilbertien (Algèbres de von Neumann), GauthierVillars, Paris, 1969.

[35] P. G. Dodds, B. de Pagter, E. M. Semenov and F. A. Sukochev Symmetric functionals and singular traces, Positivity 2 (1998), 47-75.

[36] H. Donnelly, Asymptotic expansion for the compact quotients of properly discontinuous group actions, Illinois J. Math. 23(1979), 175-221.

[37] R. G. Douglas, S. Hurder and J. Kaminker, Cyclic cocycles, renormalisation and eta-invariants, Invent. Math. 103 (1991) 101-179.

[38] T. Fack and H. Kosaki Generalised s-numbers of $\tau$-measurable operators, Pacific J. Math. 123 (1986), 269-300.

[39] K. Furutani, Fredholm Lagrangian-Grassmannian and the Maslov index, math.DG/0311495 preprint.

[40] E. Getzler, The odd Chern character in cyclic homology and spectral flow, Topology, series 32(1993), 489-507.

[41] I. C. Gohberg, M. G. Krein, Fundamental aspects of defect numbers, root numbers and indices of linear operators, Uspekhi Mat. Nauk, 12, No. 2 74(1957), 43-118.

[42] I. C. Gohberg, M. G. Krein, Introduction to the theory of non-selfadjoint operators, Translations of Mathematical Monographs, vol. 18, Amer. Math. Soc, 1969.

[43] N. Higson, The local index formula in noncommutative geometry, Contemporary Developments in Algebraic K-Theory, ICTP Lecture Notes, no. 15 (2003), 444-536.

[44] S. Hurder, Eta invariants and the odd index theorem for coverings, Contemp. Math., 105 (1990), 47-82.

[45] E. Leichtmann and P. Piazza, Spectral sections and higher Atiyah-Patodi-Singer index theory on Galois coverings, Geom. Funct. Anal. 8 (1998) 17-58.

[46] M. Lesch, On the index of the infinitesimal generator of a flow, J. Operator Theory 26 (1991), 73-92.

[47] M. Lesch, preprint, math.FA/0401411

[48] V. Mathai, $L^{2}$ invariants of covering spaces in Geometric Analysis and Lie Theory in Mathematics and Physics, Cambridge University Press, Cambridge 1998.

[49] V. Mathai, Spectral flow, eta invariants and von Neumann algebras, J. Funct. Anal., series 109 (1992), $442-456$.

[50] V. Mathai, preprint and private communication.

[51] H. McKean and I. M. Singer, Curvature and eigenvalues of the Laplacian, J. Diff. Geom., 1(1967), 43-69.

[52] R. B. Melrose, The Atiyah-Patodi-Singer index theorem, 1993, A K Peters Ltd, Wellesley, USA.

[53] R. Palais, Seminar on the Atiyah-Singer index theorem, Princeton University Press, Princeton, 1965. 
4M-T. BENAMEUR, A. L. CAREY, J. PHILliPS, A. RENNIE, F. A. SUKOCHEV, AND K. P. WOJCIECHOWSKI

[54] D. Pask and A. Rennie, The Noncommutative Geometry of Graph $C^{*}$-Algebras, J. Funct. Anal., to appear.

[55] V. S. Perera, Real valued spectral flow in a type $I I_{\infty}$ factor, Ph.D. Thesis, IUPUI, 1993 and Real valued spectral flow, Contemp. Math., 185(1995), 307-318.

[56] V. S. Perera, Real valued spectral flow in a type $I_{\infty}$ factor, Houston J. Math., 25(1999), 55-66.

[57] J. Phillips, Self-adjoint Fredholm operators and spectral flow, Canad. Math. Bull., 39(1996), 460-467.

[58] J. Phillips, Spectral flow in type I and type II factors-a new approach, Fields Institute Communications, vol. 17(1997), 137-153.

[59] J. Phillips and I. F. Raeburn An index theorem for Toeplitz operators with noncommutative symbol space, J. Funct. Anal., 120 (1993) 239-263.

[60] R. Prinzis, Traces residuelles et asymptotique du spectre d'operateurs pseudo-differentiels, Thèse, Université de Lyon, unpublished.

[61] J. Roe Elliptic operators, topology and asymptotic methods, Pitman Research Notes in Mathematics Series 395, Longman, Harlow 1998.

[62] M. A. Shubin, Pseudodifferential almost periodic operators and von Neumann algebras, Trans. Moscow Math. Soc. 1 (1979) 103-166.

[63] M. A. Shubin, Almost periodic functions and partial differential operators, Russian Math. Surveys 33:2 (1978), 1-52.

[64] M. A. Shubin, Spectral theory and index of elliptic operators with almost periodic coefficients, Russian Math. Surveys 34:2(1979), 109-157.

[65] I. M. Singer, Eigenvalues of the Laplacian and invariants of manifolds, Proceedings of the International Congress, Vancouver 1974, vol. I, 187-200.

[66] F. A. Sukochev, Unbounded Fredholm modules and submajorization, Canad. J. Math. 52(2000), 849-896.

[67] K. P. Wojciechowski, Preprint, IUPUI, Indianapolis, 1991.

UmR 7122 du CNRS, Université de Metz, Ile du Saulcy, Metz, FRAnCE, e-mail: benameur @MATH.UNIV-METZ.FR,

Mathematical Sciences Institute, Australian National University, Canberra ACT, 0200 AUSTRALIA, E-MAIL: ACAREY@MATHS.ANU.EDU.AU,

Department of Mathematics and Statistics, University of Victoria, Victoria, B.C. V8W 3P4, CANADA, E-MAIL: PHILLIPS@MATH.UVIC.CA

Institute for Mathematical Sciences, Department of Mathematics, Universitetsparken 5, DK-2100 Copenhagen DENMARK, E-MAIL: RENNiE@MATH.Ku.DK

School of Informatics and Engineering, Flinders University, Bedford Park S.A 5042 AUSTRALIA, E-MAIL: SUKOCHEV@INFOENG.FLINDERS.EDU.AU

Department of Mathematics, IUPUi (Indiana/Purdue),, Indianapolis, IN, 46202-3216, U.S.A., E-MAIL: KWOJCIECHOWSKI@MATH.IUPUI.EDU 